\title{
Alterations of Oropharyngeal Microbiome Reflected Disease Severity and Long-Term Dysbiosis in Patients With COVID-19
}

\section{Yongzhao Zhou}

Sichuan University West China Hospital

\section{Sifen Lu}

Sichuan University West China Hospital

Mengjiao Li

Sichuan University West China Hospital

\section{Minjin Wang}

Sichuan University West China Hospital

\section{Jinmin Ma}

BGI-Shenzhen: BGI Group

Yuanchen Mao

BGI-Shenzhen: BGI Group

Zhongyi Zhu

BGI-Shenzhen: BGI Group

Jiumeng Min

BGI-Shenzhen: BGI Group

Jun Xiao

Sichuan University West China Hospital

\section{Rong Qiu}

Suining Central Hospital

\section{Taibing Deng}

Guang'an People's Hospital

\section{Li Jiang}

Chuanbei Medical College: North Sichuan Medical University

\section{Jing Zhu}

Mianyang Central Hospital

\section{Yilan Zeng}

public health clinical center of Chengdu

\section{Dan Liu}

Sichuan University West China Hospital weimin li ( $\square$ weimi003@scu.edu.cn ) 
Sichuan University West China Hospital https://orcid.org/0000-0003-0985-0311

\section{Binwu Ying}

Sichuan University West China Hospital

\section{Research}

Keywords: oropharynx, microbiome, metatranscriptomics, COVID-19, dysbiosis

Posted Date: May 6th, 2021

DOI: https://doi.org/10.21203/rs.3.rs-490714/v1

License: (9) This work is licensed under a Creative Commons Attribution 4.0 International License. Read Full License 
1 Alterations of oropharyngeal microbiome reflected disease severity and long-term dysbiosis in patients with COVID-19

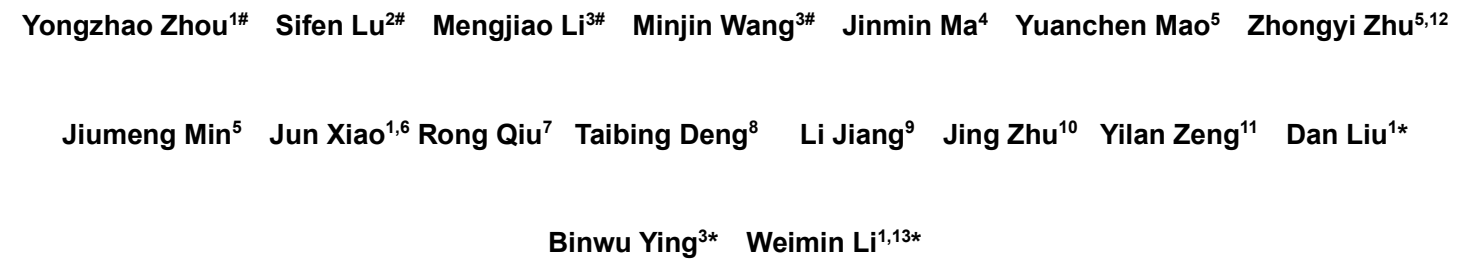


4BGI-Shenzhen, Shenzhen, 518083, China

5BGI PathoGenesis Pharmaceutical Technology, BGI-Shenzhen, Shenzhen 518083, China

6Department of Respiratory and Critical Care Medicine, People's Hospital of Ganzi Tibetan Autonomous Prefecture, Ganzi Tibetan Autonomous Prefecture 626000, China

7Department of Respiratory and Critical Care Medicine, Suining Central Hospital, Suining 629000, China

8Department of Respiratory and Critical Care Medicine, Guang'an people's Hospital, Guang'an 638000, China

9Department of Respiratory and Critical Care Medicine, Affiliated Hospital of North Sichuan Medical College, Nanchong 637000, China

10Department of Respiratory and Critical Care Medicine, Mianyang Central Hospital, Mianyang 621000, China

11Department of general medicine, Public Health Clinical Center of Chengdu, Chengdu 610041, China

12 College of Life Sciences, University of Chinese Academy of Sciences, Beijing 100049, China

13 Lead contact

\section{Abstract}

Background: Recent evidences have shown that gut microbiome of patients with COVID-19 significantly changes and can reflect the severity of the disease. And gut microbiota richness was not restored to normal levels after 6-month recovery. However, SARS-CoV-2 primarily infects the respiratory tract, few studies investigate whether the alterations of oropharyngeal microbiome is 
associated with disease severity in patients with COVID-19, and whether interferences in microbiome composition, if any, eliminate with clearance of the SARS-CoV-2 virus. We employed metatranscriptomic sequencing to analyse oropharyngeal swabs collected within a week of diagnosis COVID-19 (period of disease group: PDG) and two months after clearance of the SARS-CoV-2 virus (convalescent group: CG) from 47 patients with COVID-19. Meanwhile, oropharyngeal swabs from 40 healthy subjects were analyzed as healthy control group (HCG).

\section{Results:}

Oropharyngeal microbial composition was significantly altered in patients with COVID-19 compared with healthy controls even two months after clearance of the SARS-CoV-2 virus. Little changes in $\alpha$-diversity among HCG, PDG and CG $(P>0.05)$, but obviously changes in $\beta$-diversity among them. Notably, Prevotella increased significantly in PDG than that in HCG (Wilcoxon rank-sum test, $P<0.001$ ) and increased gradually along with the severity of patients with COVID-19 aggravated. There was a positive correlation between Prevotella and the elevation of Neutrophil percentage $(R=0.301, P=0.040)$. Similarly, SARS-CoV-2 and Aspergillus increased remarkably in critical Patients with COVID-19. There was a negative correlation between SARS-CoV-2 viral load and platelet counts $(R=-0.330, P=0.022)$.

Conclusions: The oropharyngeal microbiome in patients with COVID-19 present persistent dysbiosis even two months after clearance of the SARS- 
67 CoV-2 virus. Furthermore, alterations in oropharyngeal microbial composition 68 reflected the severity of disease in patients with COVID-19. Our findings 69 underscore that there is an urgent need to understand the specific roles of 70 oropharyngeal microorganisms in COVID-19 disease progression and 71 rehabilitation.

72 Keywords: oropharynx, microbiome, metatranscriptomics, COVID-19, dysbiosis 


\section{Background}

The COVID-19 pandemic is a once-in-a-century public health emergency, the causative pathogen was identified as a virus, named severe acute respiratory syndrome coronavirus 2 (SARS-CoV-2)[1]. Although caused only by the SARSCoV-2 virus, COVID-19 is likely affected by the rest of the microbiome as evidenced by viral persistence in the gut, and risk factors associated with gut microbial (include bacteria and fungi) dysbiosis[2-5]. Indeed, there is a study suggesting that the disease severity is concordant with composition alteration of the gut microbiome in patients with COVID-19, and the dysbiotic gut microbiota composition in patients with COVID-19 persists after clearance of the virus[6]. Besides, another study found that microbiota richness was not restored to normal levels after 6-month recovery[7]. Recently, Microbiome Centers Consortium has called for researchers try to figure out the connections between the microbiome and COVID-19[2]. SARS-CoV-2 primarily infects the respiratory tract. Study has already shown that low respiratory tract microbiota in SARS-CoV-2 infected patients were dominated with elevated levels of the upper respiratory tract (URT) commensal microbiota[8], unlike influenza virus[912], few studies have explored the relationship between SARS-CoV-2 infection and the oropharyngeal microbiome. Therefore, we investigated whether the alterations of oropharyngeal microbiome are associated with disease severity in patients with COVID-19, and whether interferences in microbiome composition, if any, eliminate with clearance of the SARS-CoV-2 virus. 


\section{Results}

112

113

114

115 122 the same timeframe. Clinical information and demographic information were

123 presented in Table 1.

\section{Study cohort}

We recruited 47 patients with COVID-19 confirmed by positive SARS-CoV-2 quantitative reverse transcription $\mathrm{PCR}(\mathrm{RT}-\mathrm{qPCR})$ to establish a longitudinal cohort study. And we collected oropharyngeal swabs at two time points (Within a week of diagnosis COVID-19 (period of disease group: PDG) and two months after clearance of the SARS-CoV-2 virus (convalescent group: CG)) during February and June 2020. The subjects were classified into mild, moderate, severe, and critical groups based on symptoms as reported before[13, 14]. As healthy controls group (HCG), we collected oropharyngeal swabs from 40 healthy human subjects free of any respiratory infections during

presented in Table 1.

Table 1 Clinical characteristics of participants

\begin{tabular}{|c|c|c|}
\hline & $\begin{array}{l}\text { COVID-19 patients } \\
\text { (period of disease group } \\
\text { / convalescent group) } *\end{array}$ & $\begin{array}{l}\text { healthy } \\
\text { controls }\end{array}$ \\
\hline Number of subjects & 47 & 40 \\
\hline $\begin{array}{l}\text { Gender } \\
\text { (females, males) }\end{array}$ & 27,20 & 20,20 \\
\hline Age, years (mean $\pm S D)$ & $44.7 \pm 15.3$ years & $\begin{array}{c}45.8 \pm 16.4 \\
\text { years }\end{array}$ \\
\hline Disease severity category & $\begin{array}{l}5 \text { mild, } 25 \text { moderate, } \\
10 \text { severe, } 7 \text { critical }\end{array}$ & NA \\
\hline \multicolumn{3}{|c|}{ Symptoms at admission, $\mathrm{n}(\%)$} \\
\hline Fever & $29(61.7 \%)$ & NA \\
\hline Cough & $29(61.7 \%)$ & NA \\
\hline Sputum & $19(40.4 \%)$ & NA \\
\hline Sore throat & $7(14.9 \%)$ & NA \\
\hline Shortness of breath & $17(36.2 \%)$ & NA \\
\hline
\end{tabular}


Received antibiotics during first week

hospitalisation by disease severity, $\mathrm{n}(\%)^{1}$

NA

Mild disease

$1(2.1 \%) \quad$ NA

moderate disease

$3(6.4 \%)$

NA

Severe disease

$5(10.5 \%)$

NA

critical disease

$7(14.8 \%)$

NA

Received antivirals during first week

hospitalisation by disease severity, $\mathrm{n}(\%)^{2}$

Mild disease

$4(8.5 \%)$

NA

moderate disease

$24(51.0 \%)$

NA

Severe disease

$10(21.3 \%)$

NA

critical disease

$7(14.8 \%)$

NA

* The 47 patients with COVID-19 were longitudinal observed in period of disease and two months after clearance of the SARS-CoV-2 virus.

${ }^{1}$ The antibiotics included quinolones and cephalosporins.

${ }^{2}$ The antivirals included Lopinavir/ritonavir, Ribavirin, Oseltamivir and interferon.

The persist dysbiosis of oropharyngeal microbiome in patients with COVID-19 even two months after clearance of the SARS-CoV-2 virus

We employed metatranscriptomic sequencing in all the 134 oropharyngeal swabs to obtain unbiased microbial profiles. In order to remove possible contamination, blank control samples, including another 6 swabs not sampled were tested. These blank control samples were applied to extract microbial genomic nucleotide and were tested using the same batch of consumables and reagents in the same equipment and laboratory. Then we analyzed the unbiased metatranscriptome sequencing data of 140 samples using a database[15] (built by updating all the complete genomes from NCBI Refseq database in real time) consisting of 4,409 genera (37,933 species) of bacteria, fungi, and viruses for taxonomic classification.

The persist oropharyngeal bacterial dysbiosis: Overall, 534 unique genera of bacteria were detected in oropharyngeal swabs from HCG, PDG and CG. 
There were 73 genera with relative abundance greater than $0.01 \%$ in 534 genera. The relative abundances of the most top20 abundant bacterial profiles in oropharyngeal swabs from HCG, PDG and CG were shown as Fig.1a, b, c, and top20 abundant bacterial profiles of every subjects were shown as Suppl.Fig.1a, b, c. Among the 534 genera, the PDG, CG and HCG groups shared with 244 genera and had 63, 36 and 61 unique genera, respectively (Fig.1e); Among the 244 shared genera, Prevotella were dominant genera of the PDG and CG (Fig.1f). Consistent with previous researches[16, 17], the major bacterial genera in HCG were Actinomyces (12.1\%), Acetobacter (10.9\%), Rothia (10.4\%), Bacteroides (9.5\%), Prevotella (8.8\%) (Fig.1a). Actinomyces and Rothia decreased remarkably in group PDG and CG (Fig.1d). Prevotella (18.3\%), Neisseria (11.0\%), Alloprevotella (5.5\%), and Fusobacterium (4.1\%) in PDG were significantly higher than that in HCG (Fig.1b and Fig.1d). Two months after SARS-CoV-2 virus has been cleared, Prevotella (19.4\%), Neisseria (14.2\%), Alloprevotella (6.9\%), and Fusobacterium $(5.1 \%)$ in CG were even higher than that in HCG or in PDG (Fig.1a, b, c and d). These results indicated that the bacterial microecology of oropharynx was obviously disturbed by SARS-CoV-2 infection, and it was hard to get back to normal even two months after the virus has been cleared.

The persist oropharyngeal fungal dysbiosis: Overall, 103 unique genera of fungi were detected in oropharyngeal swabs from HCG, PDG and CG. There were 57 genera with relative abundance greater than $0.01 \%$ in 103 genera. The 
relative abundances of the most top20 abundant fungal profiles in oropharyngeal swabs from HCG, PDG and CG were shown as Fig.2a, b, c. top20 abundant fungal profiles of every subjects were shown as Suppl.Fig.2a, b, c. The PDG, CG and HCG shared with 40 genera and had 12, 11 and 6 unique genera, respectively (Fig.2e); Among the 40 shared genera, Aspergillus were dominant genera of the PDG and CG (Fig.2f). The major genera in HCG were Malassezia (16.4\%), Aspergillus (14.8\%), Clavispora (14.1\%), Daldinia (13.6\%), Kwoniella (7.7\%) (Fig.2a). Compared with HCG, Malassezia, Clavispora and Pseudogymnoascus decreased remarkably in PDG and CG (Fig.2d). Aspergillus (20.5\%) and Saccharomyces (15.7\%) in PDG were significantly higher than that in HCG (Fig.2b, d). Two months after SARS-CoV2 virus has been cleared, Aspergillus (20.6\%), Trametes (10.6\%), Yarrowia (4.8\%), and Komagataella (3.6\%) in CG were even higher than that in HCG or PDG (Fig.2a, b, c and d). As bacterial microecology shown before, fungal microecology rarely returned to normal two months after the SARS-CoV-2 has been removed.

The persist oropharyngeal viral dysbiosis: Rhinovirus $A$, Rhinovirus $C$ and Human gamma herpesvirus 4 were the major viruses in oropharyngeal swabs from HCG. In addition to the greatly increase of SARS-CoV-2, Human beta herpesvirus 7 also increased in oropharyngeal swabs from PDG than that in HCG, but Rhinovirus $A$ and Rhinovirus $C$ decreased significantly. Opportunistic viruses, including Human gamma herpesvirus 4, Human alpha herpesvirus 1 
and Porcine type $C$ oncovirus were richer in oropharyngeal swabs from CG than that in HCG and PDG(Fig.3a).

Little changes in $\alpha$-diversity among HCG, PDG and CG: We investigated longitudinal dynamics of oropharynx microbial (include bacteria and fungi) profiles in COVID-19 over time of exacerbation and explored whether recovery from SARS-CoV-2 infection was associated with restoration of oropharyngeal microbial profiles to a community similar to that of healthy individuals. Results from $\alpha$-diversity microbial analyses (measured by the Shannon Diversity Index and Observed Diversity Index) have demonstrated little changes in $\alpha$-diversity among PDG, CG and HCG $(P>0.05)$ (Fig.4a, b).

Obviously changes in $\beta$-diversity among HCG, PDG and CG: Principal coordinates analysis (PCOA) based on Bray-Curtis distance was used to visualize the apparent differences in microbial community structure. Fig. 4c shows that the PDG and CG are extremely separate from HCG, suggesting that oropharyngeal microbial structures were modulated by SARS-CoV-2 infection and could not recover even virus was cleared two month later. The analysis of similarities (ANOSIM) plot shows that the inter-group difference was greater than the intra-group difference (Fig. $\mathbf{4 d}, \mathrm{R}=0.246, P=0.001$ ).

In conclusion, the SARS-CoV-2 infection has a strong influence on the composition of oropharyngeal microbiome.

Obviously changes in antibiotic resistance gene expression among HCG, PDG and CG: To characterize antibiotic resistance gene expression profiles, 
we analyzed the metatranscriptomic sequence reads based on Comprehensive

211 Antibiotic Resistance Database (CARD 2020; https://card.mcmaster.ca).

212 Across the dataset, we identified genes that confer resistance to 13 classes of

213 antibiotics (Fig. 5a, b) with variations across the swabs. The expression of 214 multidrug resistance genes (especially multidrug resistance, tetracycline and 215 glycopeptide) in the oropharyngeal microbiome of patients with COVID-19 were 216 higher than that in healthy controls, and returned to normal two months after 217 virus clearance.

Alteration of main oropharyngeal microbial composition reflects disease

220 In order to explore the role of oropharyngeal microbiome in patients with

221 COVID-19, PDG subjects were further categorized as mild, moderate, severe, 222 and critical according to the Diagnosis and Treatment Scheme for COVID-19 released by the National Health Commission of China (Version7)[8, 13, 14], and

224 mild, moderate patients with COVID-19 were divided as mild-moderate group microbial composition in patients with COVID-19 and the magnitude of COVID-

22819 severity were analysed.

Prevotella increased gradually along with the symptom aggravated: Top20 rank-sum test, $p<0.001$ ) ( Fig.1d). As to the subgroup of PDG, Top20 bacterial 
profiles in oropharyngeal swabs from MMG and SCG were shown as Fig.6a, b. The abundance of Prevotella in SCG was significantly higher (20.1\%) than that in MMG (17.3\%) (Fig.6c). Prevotella increased gradually, along with the severity of patients with COVID-19 aggravated gradually. (Fig.6d). To investigate the potential effects of the Prevotella in oropharynx of patients with COVID-19, we used a spearman analysis strategy for the parallel evaluation of all quantitative clinical traits from COVID-19 patients. Remarkably, there was a positive correlation between Prevotella and the elevation of Neutrophil percentage $(R=0.301, p=0.040)$ (Fig.6e). These results indicated that it would be necessary to verify the synergic role of Prevotella in inducing severity of SARS-CoV-2 mediated pneumonia.

Aspergillus increased remarkably in critical Patients with COVID-19: It was observed that patients with severe COVID-19 had an increased risk of coexisting Aspergillus infection [18]. As to the subgroup of PDG, Top20 fungal profiles in oropharyngeal swabs specimens from MMG and SCG were shown as Fig.7a, b. The abundance of Aspergillus in SCG was significantly higher $(21.7 \%)$ than that in MMG (19.8\%) (Fig.7c). In particularly, Aspergillus increased remarkably in critical Patients with COVID-19 (Fig.7d). Therefore, monitoring the change in abundance of Aspergillus in oropharynx of COVID-19 patients should be considered[18, 19].

Decreased $\alpha$-diversity appeared to be associated with COVID-19 severity: The $\alpha$-diversity of oropharyngeal microbial profiles did not differ between 
healthy controls and patients with COVID-19 (Fig. 4a,b, $P>0.05)$. However, the diversity and richness of oropharyngeal microbial profiles were both significantly lower in SCG group than MMG group. Obviously, the $\alpha$-diversity decreased gradually along with the symptom aggravated(Fig. 8a,b, $P<0.01$ ). Besides, Principal coordinates analysis (PCoA) of $\beta$-diversity between SCG and MMG demonstrated that the microbial cluster away from each other (Fig.8c), there were also significant differences among mild, moderate, severe, and critical groups (Fig.8d).

The severity of COVID-19 was positively correlated with higher SARSCoV-2 viral load: Fig.3b and Fig.3c showed that severe and critical patients with COVID-19 had much higher SARS-CoV-2 viral load than others. There was a negative correlation between SARS-CoV-2 viral load and platelet counts $(R=$ $-0.330, P=0.022)$ (Fig.3d). These results indicated that SARS-CoV-2 viral load may be a biomarker to evaluate if they are able to predict lower platelet counts and correlate with the severity of COVID-19 disease. In conclusion, early check of SARS-CoV-2 viral load by metatranscriptome may be helpful for early detection of critically COVID-19 patients.

\section{Discussion}

SARS-COV-2 and other viral infections may alter the microbial composition of the upper respiratory tract, foster enhanced growth of pathogens, and facilitate the subsequent entry of large microbial loads into the lower respiratory tract [8, $12,20,21]$, which are frequently associated with a more severe clinical 
course[12, 18, 19, 22-24]. Besides, a specific bacterial formulation has showed a significant ameliorating impact on the clinical conditions of patients positive for SARS-CoV-2 infection[25]. And given the unequivocal association between gut microbiota composition and COVID-19 disease severity, there is a pressing need to better understand how perturbation of the host oropharyngeal microbiome correlates with SARS-COV-2 infections[6]. Based on analysis unbiased metatranscriptomic data, our longitudinal study has firstly confirmed that oropharyngeal microbiome composition reflects disease severity and presents long-term dysbiosis in patients with COVID-19.

The bacterial and fungal microecology of oropharynx were significantly perturbed by SARS-CoV-2 infection, and they were even hard to return back to normal two months after the virus was cleared. Certainly, it is hard to ignore the influence of antibiotic exposure on URT microbiome. However, recent proof demonstrated that antibiotic drugs have short-term influence on the human gut microbiome with the bacterial community largely recovering within 30 days of antibiotic treatment[26, 27]. So, we speculate that SARS-COV-2 infection is the key factor affecting the microecological dynamics of oropharynx. As other viral infections, antiviral immune responses induced by SARS-COV-2 is associated with changes in microbial composition and function in the URT, which in turn may alter immune function of URT, thereby enhancing the sustainable proliferation of some bacterial species[12, 21].

Our study conducted in healthy subjects and who underwent SARS-COV-2 
infection failed to demonstrate obvious changes in $\alpha$-diversity of the oropharyngeal microbiome. Thus, as other viral infections[12], the effects of SARS-COV-2 infection on diversity itself are not presently considered a good indicator of risk for complications, including secondary bacterial pneumonias. The effect of SARS-COV-2 infection on $\alpha$ diversity is variable and is not currently considered a good indicator of the viral infection. Nevertheless, our study in patients with SARS-COV-2 demonstrated that the $\alpha$-diversity of oropharyngeal microbiome decreased significantly associated with COVID-19 severity.

In recent studies by next generation sequencing and MinION sequencing, Prevotella was found to be the common bacteria in URT of patients with COVID19 [28, 29]. Notably, rich Prevotella was also detected in the intestines of patients with COVID-19[3]. The synergism of Prevotella in causing the severity of streptococcus pneumoniae mediated pneumonia was already known[30]. Our data about patients with COVID-19 further demonstrated that Prevotella in oropharynx increased gradually consist with the symptom aggravated and could not return back to normal even two months after the SARS-COV-2 was cleared. Through microaspiration from URT, Prevotella could participate in the immune homeostasis of the low respiratory tract [31], and pulmonary inflammation had been associated with accumulation of the pulmonary microbiome with Prevotella, this bacterium mainly activated Toll-like receptor 2 and enhanced the expression IL-23 and IL-1 [32]. In vitro, Prevotella could 
stimulate the production of IL-8, IL-6, and CCL20 in lung epithelial cells, which promoted neutrophil recruitment [33]. In vivo, we also confirmed that there was a positive correlation between Prevotella and the elevation of Neutrophil percentage. Latest evidence about host-pathogen interactions in the severity of COVID-19 also confirmed Prevotella proteins were over expressed in patients with COVID-19, and Prevotella proteins, but not SARS-COV-2 proteins were involved in multiple interactions with NF-kB, which was involved in increasing clinical severity of COVID-19[34]. In conclusion, Prevotella may play an important role in COVID-19 outbreak and should be given attention for understanding disease mechanisms and improving treatment outcomes.

Aspergillus is a common fungal pathogen of secondary infection in patients with critical COVID-19[18, 19, 35, 36]. Our results showed that Aspergillus increased remarkably in oropharynx of critical Patients with COVID-19. The respiratory tract is an interconnected system consisting of oropharynx and low respiratory tract, with microaspiration serving as the primary route of Aspergillus immigration from the oropharynx to the low respiratory tract. Therefore, for early detection of secondary fungal infections, monitoring the change in abundance of Aspergillus in oropharynx of patients with COVID-19 should be considered.

Soon after the onset of the global SARS-CoV-2 epidemic, the presence of other common respiratory viruses declined and even disappeared[22, 28]. Our results by metatranscriptomics are consistent with the absence of other common respiratory virus coinfection in patients with COVID-19. More 
importantly, the severity of COVID-19 was positively correlated with higher SARS-CoV-2 viral load and opportunistic viruses were rich even fully recovered two months later, and there was a negative correlation between SARS-CoV-2 viral load and platelet counts. Platelet count showed significantly lower levels in severe patients compared to non-severe patients[37]. Meta-analysis of 1799 patients also revealed those with severe COVID-19 infections had significantly lower platelet counts $[37,38]$.Therefore, besides keeping an eye on opportunistic viral infection of convalescent patients with COVID-19, early check of SARS-CoV-2 viral load by metatranscriptome may be helpful for early detection of critically COVID-19 patients.

More importantly, we firstly performed metatranscriptomics to characterize the abundance and diversity of antibiotic resistance genes in the oropharynx microbiome of patients with COVID-19. Higher relative abundance of antibiotic resistance genes in the oropharyngeal microbiome of patients with COVID-19 were decreased to normal two months after virus clearance. In line with previous study[26], Characterization of bacterial antibiotic resistance genes in the URT microbiome of patients with COVID-19 might help better to choose the appropriate antibiotics for bacterial co-infection and secondary infection in patients with COVID-19.

\section{Conclusions}

SARS-CoV-2 primarily infects the respiratory tract and significantly perturbs microecology of oropharynx. Alterations in oropharyngeal microbial 
composition, particularly of Prevotella and Aspergillus, reflect the severity of

365 disease in patients with COVID-19. Furthermore, the oropharyngeal

366 microbiome in patients with COVID-19 present persistent dysbiosis two months

367 after clearance of the SARS-CoV-2 virus. These findings underscore an urgent

368 need to understand the specific roles of oropharyngeal microorganisms in

369 COVID-19 disease progression and rehabilitation as well as co-infection and

370 secondary infection.

\section{Abbreviations}

372 SARS-CoV-2: severe acute respiratory syndrome coronavirus 2

373 COVID-19: coronavirus disease 2019

374 URT: upper respiratory tract

375 PDG: period of disease group

376 CG: convalescent group

377 HCG: healthy controls group

378 RT-qPCR: quantitative reverse transcription polymerase chain reaction

\section{Methods}

\section{Study design and specimens collect}

381 We recruited 47 patients with COVID-19 confirmed by positive SARS-CoV-2

382 quantitative reverse transcription $\mathrm{PCR}(\mathrm{RT}-\mathrm{qPCR})$ to establish a longitudinal 383 cohort study. The severity status of confirmed COVID-19 cases was 384 categorized as mild, moderate, severe, and critical according to the Diagnosis and Treatment Scheme for COVID-19 released by the National Health 
Commission of China (Version 7). As healthy controls group, we recruited 40 healthy human subjects free of any respiratory infections during the same timeframe. Written consents were obtained from patients or their guardian(s).

Clinical information and demographic information were collected. Oropharyngeal samples were obtained for routine diagnostic purposes, and the residual clinical diagnostic samples were provided for research and surveillance purposes. We collected oropharyngeal swabs at two time points (Within a week of diagnosis COVID-19 and two months after clearance of the SARS-CoV-2 virus. During the same timeframe, we collected oropharyngeal swabs from 40 healthy human subjects free of any respiratory infections. In order to remove possible contamination, blank control samples, including another 6 swabs not sampled, were collected. After collection, the samples were placed into a tube containing $2 \mathrm{~mL}$ viral RNA preservation solution.

\section{Samples processing and quantitative real-time PCR test for COVID-19}

Total RNA was extracted using QIAamp viral RNA mini kit (QIAGEN 52904, Germany) following the manufacturer's protocol.

Real-time RT-PCR was performed by amplifying two target genes, including open reading frame 1ab (ORF1ab) and nucleocapsid protein (N) using RT-PCR kit (Sansure Biotech Inc, China) on a Real-time PCR thermal cycler (ABI 7500 system, Applied Biosystems instruments, USA).

\section{Metatranscriptomic sequencing}

After extraction, the first strand cDNA was prepared by priming with random 
hexamers followed. Standard RNAseq library was constructed using NGS library construction kit (Genskey 1906). The libraries were sequenced on Illumina NextSeq 500-sequencer using a 75-cycle single-end sequencing strategy and each sample will get nearly 20 million reads.

\section{Data processing}

First, software fastp (parameters: -q $15-\mathrm{u} 40$ - I50, version: 0.19.5) [39] was used to filter low-quality reads, remove adapter and Komplexity (parameters: t 0.4, version: Nov-2019)[40]was used to remove low complexity reads for raw data. Then, filtered reads were mapped to the ensembl 84 (GRCh38) human reference genome to remove host sequences using HISAT2 (version 2.1.0) with default parameters [41]. Next, unmapped reads were annotated taxonomic classifications by Kraken2 (version 2.0.9, parameters: --threads 24 -confidence 0.1)[42]with a self-build database (built by downloading all the complete genomes from NCBI Refseq database, including the SARS-COV-2 reference NC_045512.2. And only genomes from archaea, bacteria, fungi, protozoa and viral were selected for building a classification database for Kraken2 $(k=35, \ell=31))$. Then, abundance of taxonomy was estimated using Bracken (version 2.5, parameters: -r 75 -I G, S -t 0) [43]. Finally, microorganisms satisfying the following criteria were retained for the subsequent analysis: (1) archaea, bacteria, fungi, or virus; (2) 10-fold higher filtered RPM than that in the negative control (NC); (3) no batch effect; (4) no known contamination;(5) only human virus. 
In order to obtain information of antibiotic resistance gene. First, unmapped reads were assemblied using SPAdes (version: 3.13.0) with default parameters [44] and scaffolds less than 150 bp were removed. Then, MetaGeneMark [45] was used to predict protein coding genes from the above filtered scaffolds. Next, the abundance of antibiotic resistance genes were annotated by aligning the sequence of the above predicted genes to Comprehensive Antibiotic Resistance Database (CARD 2020; https://card. mcmaster.ca) [46] using the HISAT2 alignment method with default parameters. Each gene was filtered to have at least two matching reads. Finally, only antibiotic resistance genes occurred in $>25 \%$ of samples were used for further statistical analyses.

\section{Statistical analysis}

Statistical analyses were done using $\mathrm{R}$ (version 3.5.1). The $\alpha$-diversity was analyzed by vegan function with $\mathrm{R}$ packages. Principal co-ordinates analysis (PCoA) was based on Bray-Curtis dissimilarity (BCD) distance matrices[47]. Differences between groups in the $\alpha$-diversity were evaluated using the MannWhitney test or the Kruskal-Wallis test. False discovery rate (FDR) values were estimated using the Benjamini-Yekutieli method to control for multiple testing [48]. Principal component analysis (PCA) was analyzed by prcomp function with R packages [49]. Analysis of similarities (ANOSIM) was used to statistically test whether there is a significant difference between two or more groups of sampling units[50]. The $\log 10$ value was used to show the abundance of antibiotic resistance gene. Finally, the spearman correlation method [51] was 
452 453 and a correlation coefficient of more than 0.300 is considered [52]. A p-value of $454<0.05$ was considered statistically significant. Concerning symbols used to 455 represent higher orders of significance, $P<0.05$ was indicated by *,$P<0.01$ by 456

used to analyze the correlation analysis between microbe and clinical index, ** and $P<0.001$ by ***.

\section{Acknowledgements}

We thank Yannan Lini for assistance with processing language editing of the manuscript. We thank Genskey for their assistance in the sequencing work.

\section{Authors' contributions}

WML, BWY, DL, YZZ, MJL and MJW contributed substantially to the conception and design of the study and interpretation and wrote the manuscript. RQ, TBD, LJ, JZ and YLL undertook clinical sample collection and processing and analyses of clinical records. YZZ, SFL, MJL, MJW, JMM, YCM, ZYZ, JMM and JX contributed substantially to the acquisition of data and the analysis. YZZ and SFL contributed substantially to data interpretation. The authors read and approved the final manuscript.

\section{Funding}

This work was funded by the Sichuan Provincial Program for Diagnostic and Treatment of COVID-19 (2020YFS0002, 2020YFS0001, 2020YFS0572).

\section{Availability of data and materials}

The sequencing data from this study have been deposited in the CNSA (https:// db.cngb.org/cnsa/) of CNGBdb with accession number CNP0001393. 
475 This study was approved by The Biomedical Research Ethics Committee of

476 West China Hospital and the document IDs are NO.2020(100), NO.2020 (193)

477 and NO.2020 (267). Written consents were obtained from patients or their 478 guardian(s). All work was performed in accordance with the Helsinki 479 Declaration.

\section{Consent for publication}

481 Not applicable.

\section{Competing interests}

483 The authors declare that they have not competing interests.

\section{$484 \quad$ References}

485 1. Zhu N, Zhang D, Wang W, Li X, Yang B, Song J, Zhao X, Huang B, Shi W, Lu R et al: A Novel Coronavirus from Patients with Pneumonia in China, 2019. N Engl J Med 2020, 382(8):727733.

2. Microbiome Centers Consortium CC: Coordinating and Assisting Research at the SARS-CoV2/Microbiome Nexus. mSystems 2020, 5(6).

3. Tao W, Wang X, Zhang G, Guo M, Ma H, Zhao D, Sun Y, He J, Liu L, Zhang K et al: Re-detectable positive SARS-CoV-2 RNA tests in patients who recovered from COVID-19 with intestinal infection. Protein Cell 2020. Alterations in Gut Microbiota of Patients With CoVID-19 During Time of Hospitalization. 
5. Zuo T, Zhan H, Zhang F, Liu Q, Tso EYK, Lui GCY, Chen N, Li A, Lu W, Chan FKL et al: Alterations in Fecal Fungal Microbiome of Patients With COVID-19 During Time of Hospitalization until Discharge. Gastroenterology 2020, 159(4):1302-1310 e1305.

6. Yeoh YK, Zuo T, Lui CY, Zhang F, Ng SC: Gut microbiota composition reflects disease severity and dysfunctional immune responses in patients with COVID-19. Gut 2021:gutjnl-2020323020.

7. Chen Y, Gu S, Chen Y, Lu H, Shi D, Guo J, Wu WR, Yang Y, Li Y, Xu KJ et al: Six-month follow-up of gut microbiota richness in patients with COVID-19. Gut 2021.

8. Shen Z, Xiao Y, Kang L, Ma W, Shi L, Zhang L, Zhou Z, Yang J, Zhong J, Yang D et al: Genomic Diversity of Severe Acute Respiratory Syndrome-Coronavirus 2 in Patients With Coronavirus Disease 2019. Clin Infect Dis 2020, 71(15):713-720.

9. Qin T, Geng T, Zhou H, Han Y, Ren H, Qiu Z, Nie X, Du T, Liang J, Du P et al: Super-dominant pathobiontic bacteria in the nasopharyngeal microbiota as causative agents of secondary bacterial infection in influenza patients. Emerg Microbes Infect 2020, 9(1):605-615.

10. Martin-Loeches I, M JS, Vincent JL, Alvarez-Lerma F, Bos LD, Sole-Violan J, Torres A, Rodriguez A: Increased incidence of co-infection in critically ill patients with influenza. Intensive Care Med 2017, 43(1):48-58.

11. Kaul D, Rathnasinghe R, Ferres M, Tan GS, Barrera A, Pickett BE, Methe BA, Das SR, Budnik I, Halpin RA et al: Microbiome disturbance and resilience dynamics of the upper respiratory tract during influenza A virus infection. Nat Commun 2020, 11(1):2537.

12. Hanada S, Pirzadeh M, Carver KY, Deng JC: Respiratory Viral Infection-Induced Microbiome Alterations and Secondary Bacterial Pneumonia. Front Immunol 2018, 9:2640. 
13. Jian W, Jun L, Xinguo Z, Chengyuan L, Wei W, Dawei W, Wei X, Chunyu Z, Jiong Y, Bin J: Clinical Characteristics of Imported Cases of Coronavirus Disease 2019 (COVID-19) in Jiangsu Province: A Multicenter Descriptive Study. Clinical Infectious Diseases.

14. Lu J, du Plessis L, Liu Z, Hill V, Kang M, Lin H, Sun J, Francois S, Kraemer MUG, Faria NR et al: Genomic Epidemiology of SARS-CoV-2 in Guangdong Province, China. Cell 2020, 181(5):9971003 e1009.

15. Zhang H, Ai JW, Yang W, Zhou X, Zhang W: Metatranscriptomic Characterization of COVID-19 Identified A Host Transcriptional Classifier Associated With Immune Signaling. Clinical Infectious Diseases 2020.

16. The nasal cavity microbiota of healthy adults. Microbiome 2014, 2(1):1-5.

17. Lemon KP, Klepac-Ceraj V, Schiffer HK, Brodie EL, Lynch SV, Kolter R: Comparative analyses of the bacterial microbiota of the human nostril and oropharynx. mBio 2010, 1(3).

18. Koehler P, Cornely OA, Bottiger BW, Dusse F, Eichenauer DA, Fuchs F, Hallek M, Jung N, Klein F, Persigehl T et al: COVID-19 associated pulmonary aspergillosis. Mycoses 2020, 63(6):528-534.

19. Gangneux JP, Bougnoux ME, Dannaoui E, Cornet M, Zahar JR: Invasive fungal diseases during COVID-19: We should be prepared. J Mycol Med 2020, 30(2):100971.

20. Wolter N, Tempia S, Cohen C, Madhi SA, Venter M, Moyes J, Walaza S, Malope-Kgokong B, Groome M, du Plessis M et al: High nasopharyngeal pneumococcal density, increased by viral coinfection, is associated with invasive pneumococcal pneumonia. J Infect Dis 2014, 210(10):1649-1657.

21. Haocheng Z, Jing-Wen A, Wenjiao Y, Xian Z, Fusheng H, Shumei X, Weiqi Z, Yang L, Yiqi Y, Xuejing G: Metatranscriptomic Characterization of COVID-19 Identified A Host Transcriptional 
22. Calcagno A, Ghisetti V, Burdino E, Trunfio M, Allice T, Boglione L, Bonora S, Di Perri G:

24. Kim D, Quinn J, Pinsky B, Shah NH, Brown I: Rates of Co-infection Between SARS-CoV-2 and review and meta-analysis. Clin Microbiol Infect 2020.

25. d'Ettorre G, Ceccarelli G, Marazzato M, Campagna G, Pinacchio C, Alessandri F, Ruberto F, Rossi

G, Celani L, Scagnolari C et al: Challenges in the Management of SARS-CoV2 Infection: The

27. MacPherson CW, Mathieu O, Tremblay J, Champagne J, Nantel A, Girard SA, Tompkins TA: Gut 
29. Moore SC, Penrice-Randal R, Alruwaili M, Randle N, Armstrong S, Hartley C, Haldenby S, Dong $\mathrm{X}$, Alrezaihi A, Almsaud M et al: Amplicon-Based Detection and Sequencing of SARS-CoV-2 in Nasopharyngeal Swabs from Patients With COVID-19 and Identification of Deletions in the Viral Genome That Encode Proteins Involved in Interferon Antagonism. Viruses 2020, 12(10).

30. Nagaoka K, Yanagihara K, Morinaga Y, Nakamura S, Harada T, Hasegawa H, Izumikawa K, Ishimatsu Y, Kakeya H, Nishimura M: Prevotella intermedia Induces Severe Bacteremic Pneumococcal Pneumonia in Mice with Upregulated Platelet-Activating Factor Receptor Expression. Infection and Immunity 2014, 82(2):587-593.

31. Huffnagle $\mathrm{GB}$, Dickson $\mathrm{RP}$, Lukacs NW: The respiratory tract microbiome and lung inflammation: a two-way street. Mucosal Immunol 2017, 10(2):299-306.

32. Segal LN, Alekseyenko AV, Clemente JC, Kulkarni R, Wu B, Chen H, Berger KI, Goldring RM, Rom WN, Blaser MJ: Enrichment of lung microbiome with supraglottic taxa is associated with increased pulmonary inflammation. Microbiome 2013, 1.

33. Larsen, Madura J: The immune response to Prevotella bacteria in chronic inflammatory disease. Immunology 2017, 151(4).

34. Khan AA, Khan Z: COVID-2019-associated overexpressed Prevotella proteins mediated hostpathogen interactions and their role in coronavirus outbreak. Bioinformatics 2020, 36(13):4065-4069.

35. Vaillancourt M, Jorth P: The Unrecognized Threat of Secondary Bacterial Infections with COVID-19. mBio 2020, 11(4).

36. Biesen SV, Kwa D, Bosman RJ, Juffermans NP: Detection of Invasive Pulmonary Aspergillosis in COVID-19 with Non-directed Bronchoalveolar Lavage. American Journal of Respiratory and 
37. Kermali M, Khalsa RK, Pillai K, Ismail Z, Harky A: The role of biomarkers in diagnosis of COVID-

38. Lippi G, Plebani M, Henry BM: Thrombocytopenia is associated with severe coronavirus disease 2019 (COVID-19) infections: A meta-analysis. Clinica Chimica Acta 2020.

39. Chen S, Zhou Y, Chen Y, Gu J: fastp: an ultra-fast all-in-one FASTQ preprocessor. Bioinformatics 2018, 34(17):i884-i890.

40. Zijie S, Yan X, Lu K, Wentai M, Leisheng S, Li Z, Zhuo Z, Jing Y, Jiaxin Z, Donghong Y: Genomic

44. Bankevich A, Nurk S, Antipov D, Gurevich AA, Dvorkin M, Kulikov AS, Lesin VM, Nikolenko SI, 

AA, Sihan L: CARD 2020: antibiotic resistome surveillance with the comprehensive antibiotic resistance database. Nucleic Acids Research.

47. Kristi B, Brett WM, Sharon WT, Martin M, Mia J, Melissa Z, Taylor MW, Douglas RG: Differentially Regulated Host Proteins Associated with Chronic Rhinosinusitis Are Correlated with the Sinonasal Microbiome. Frontiers in Cellular and Infection Microbiology 2017, 7:504-.

48. Benjamini Y, Yekutieli D: The control of the false discovery rate in multiple testing under dependency. Annals of Statistics 2001, 29(4).

49. Dubey G, Kollah B, Gour VK, Shukla AK, Mohanty SR: Diversity of bacteria and archaea in the rhizosphere of bioenergy crop Jatropha curcas. Biotech 2016, 6(2):257.

50. Marti, J., Anderson, Daniel, C., I., Walsh: PERMANOVA, ANOSIM, and the Mantel test in the face of heterogeneous dispersions: What null hypothesis are you testing? Ecological Monographs 2013.

51. De Winter JCF, Gosling SD, Potter J: Comparing the Pearson and Spearman Correlation Coefficients Across Distributions and Sample Sizes: A Tutorial Using Simulations and Empirical Data. Psychological Methods 2016, 21(3):273-290.

52. Mukaka M: Statistics corner: A guide to appropriate use of correlation coefficient in medical research. Malawi Medical Journal 2012, 24(3):69-71. 
a

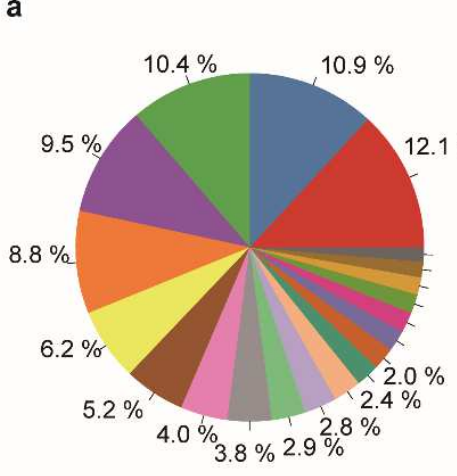

c

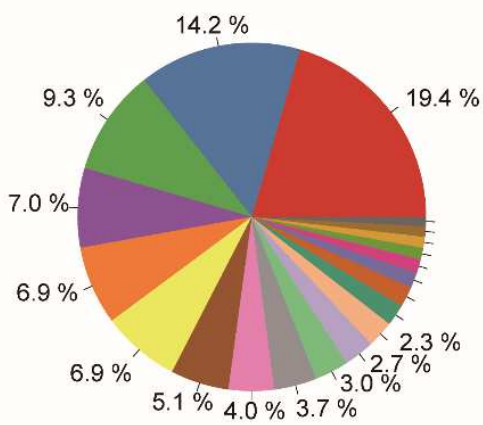

- Actinomyces

- Acetobacter

- Rothia

- Bacteroides

- Prevotella

Dolosigranulum

- Neisseria

- Veillonella

- Primorskyibacter

- Schaalia

Alloprevotella

Kytococcus

- Porphyromonas

- Mycoplasma

- Capnocytophaga

- Fusobacterium

- Campylobacte

- Gemella

- Atopobium

- Prevotella

- Neisseria

- Bacteroides

Alloprevotella

Primorskyibacte

- Fusobacterium

- Campylobacter

- Porphyromonas

- Actinomyces

- Capnocytophaga

Veillonella

- Treponema

- Komagataeibacter

- Selenomonas

- Tannerella

- Lautropia

- Parabacteroides

- Pluralibacter

- Glaesserella

Neisseria

Actinomyces

Acetobacter

Rothia

Alloprevotella

Porphyromonas

- Fusobacterium

Primorskyibacter

- Veillonella

Capnocytophaga

- Campylobacter

- Dolosigranulum

- Treponema

- Schaalia

- Lautropia

Kytococcus

Gemella

Selenomonas

$$
\text { CG }
$$

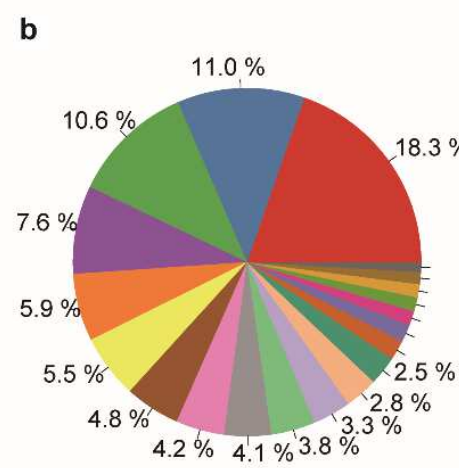

- Prevotella

- Neisseria

- Bacteroides

- Rothia

Porphyromonas

Alloprevotella

Actinomyces

Capnocytophaga

- Fusobacterium

- Veillonella

Treponema

Campylobacter

- Lautropia

Acetobacter

- Gemella

- Selenomonas

- Parabacteroides

- Schaalia

- Glaesserella

d

- Kytococcus

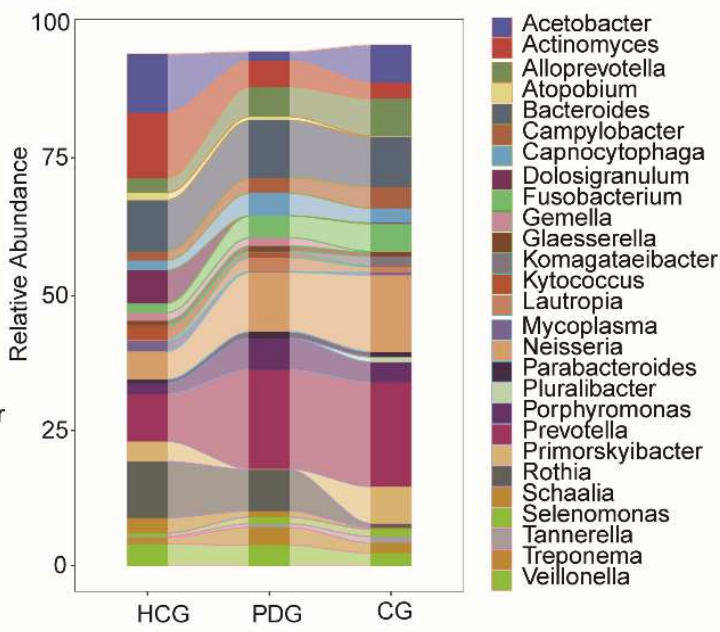

e

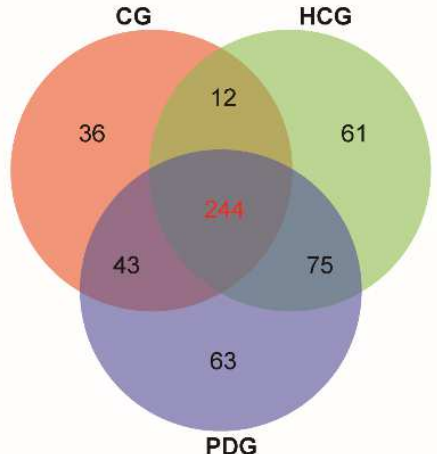

Fig.1 Oropharyngeal bacterial characterization and alterations among HCG, 
629 than that in HCG (Wilcoxon rank-sum test, $p<0.001$ ). e Venn diagram showing 630 shared and unique genus of the three groups. $f$ Ternary plots depicting the 244 631 shared bacterial landscape on genus level among HCG, PDG and CG group. 632 The sum of the proportion for one specific bacteria in the three groups was set 633 as 1 , and the proportion of one specific bacteria in each group is equal to its 634 corresponding relative abundance divided by the relative abundance sum of 635 this bacteria in the three groups. The size of the circles represents the relative 636 abundance of the genus. 


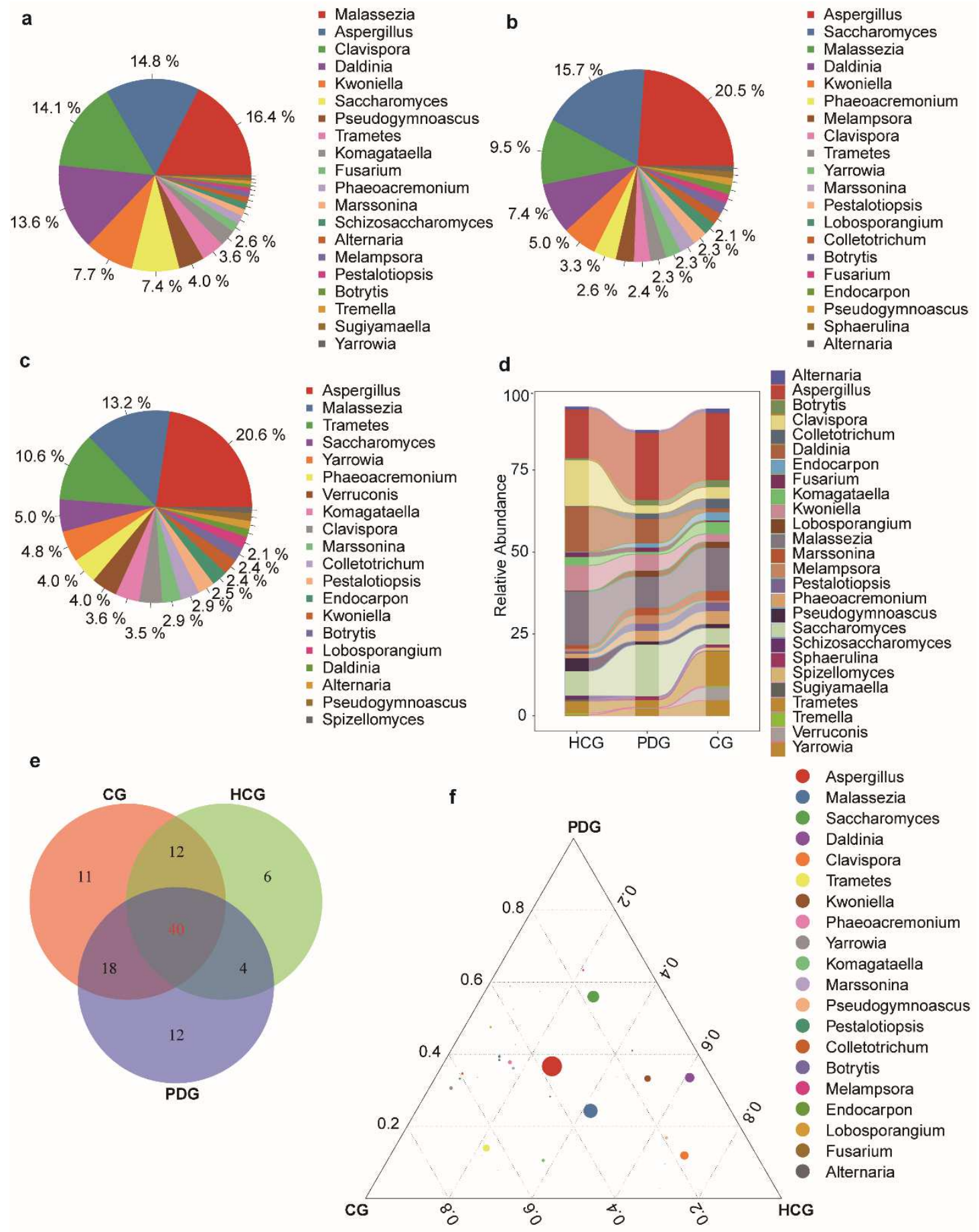

Fig.2 Oropharyngeal fungal characterization and alterations among HCG, PDG

CG. $d$ Alterations of top20 fungal communities among the three groups at the 
643

644

645

646

647

648

649

650

651

652

653

654

groups. f Ternary plots depicting the 244 shared fungal landscape on genus level among HCG, PDG and CG group. The sum of the proportion for one specific bacteria in the three groups was set as 1, and the proportion of one specific fungi in each group is equal to its corresponding relative abundance divided by the relative abundance sum of this fungi in the three groups. The size of the circles represents the relative abundance of the genus.
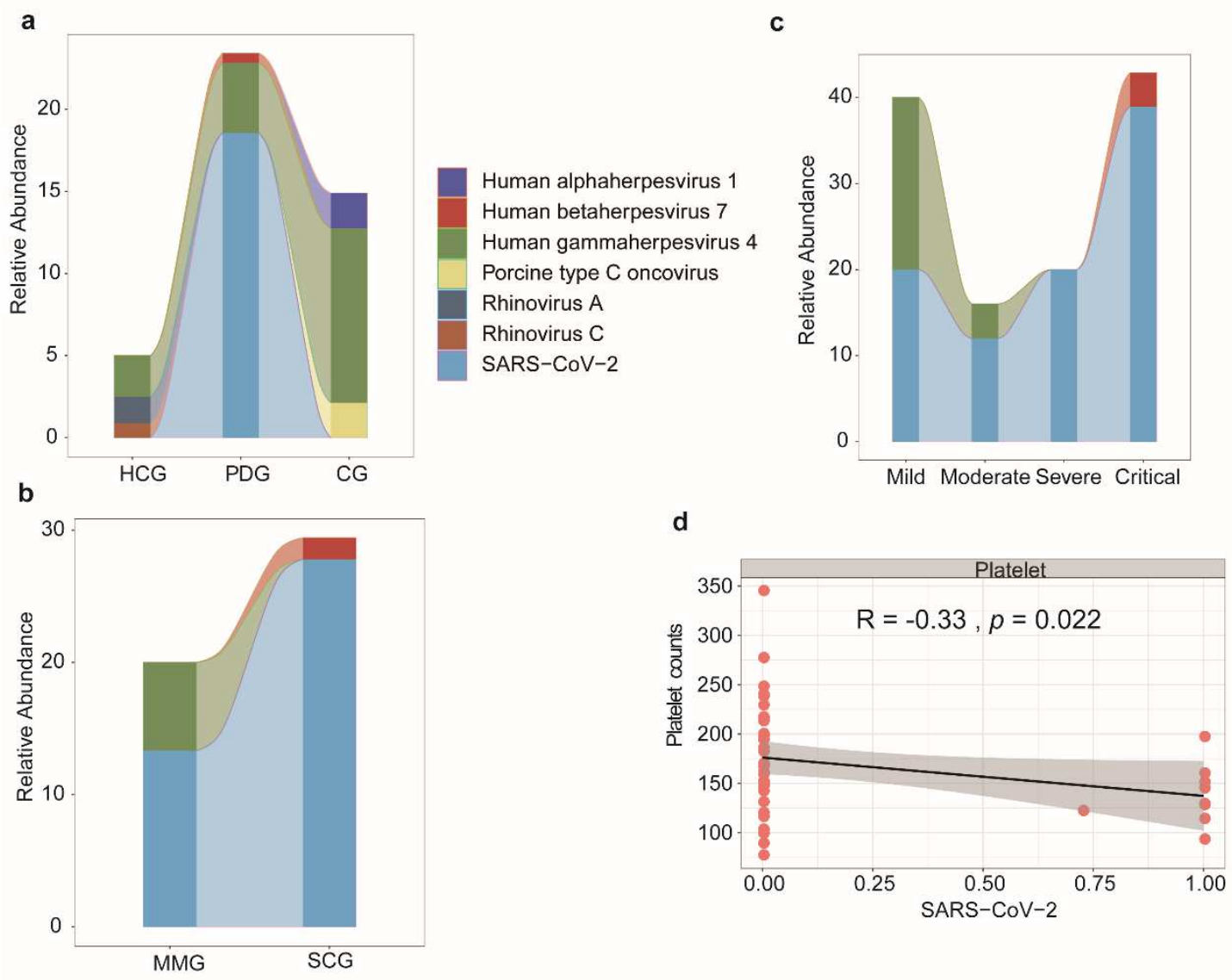

Fig.3 Oropharyngeal SARS-CoV-2 viral load was positively correlated with the severity of COVID-19. a The relative abundance changes of 7 oropharyngeal viral species (including SARS-COR-2) among HCG, PDG and CG group, b among MMG and SCG group, c among Mild, Moderate, Severe and Critical group. d Negative correlation between SARS-CoV-2 viral load (x-axis) and 
655

656

657

a

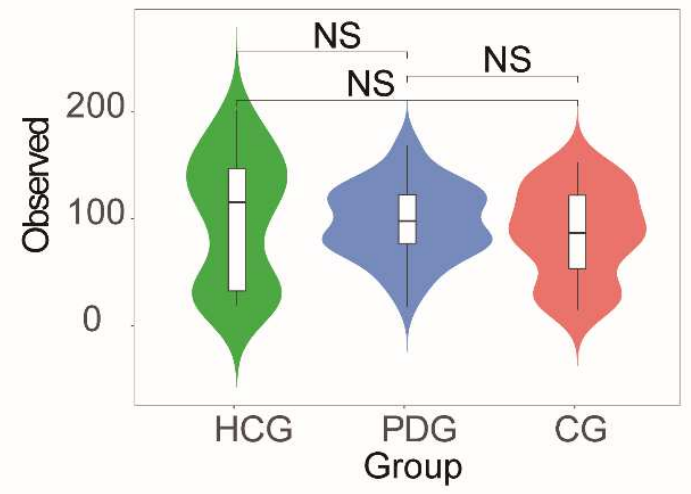

C

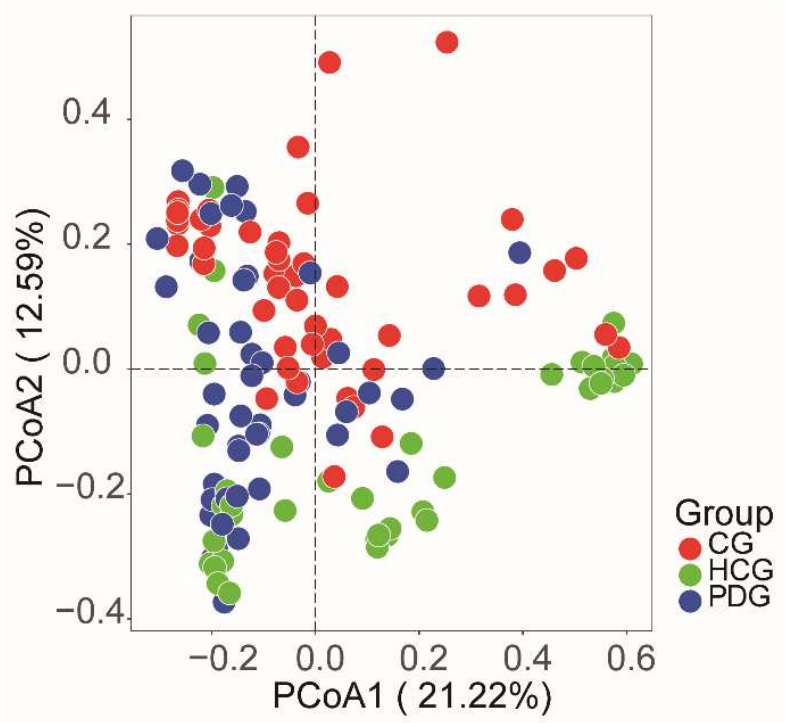

b

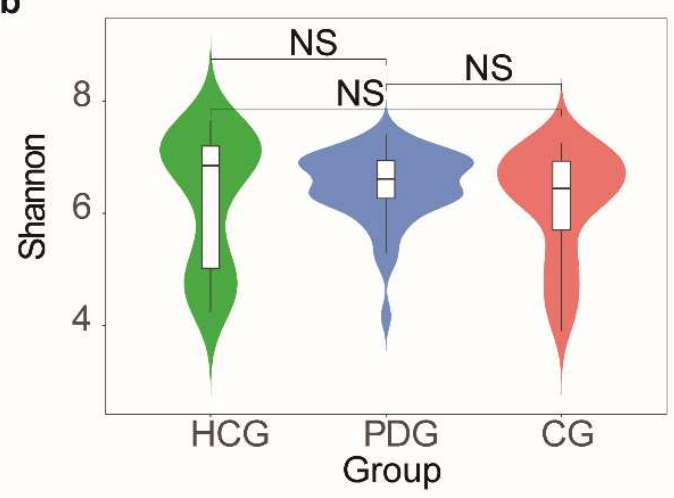

d

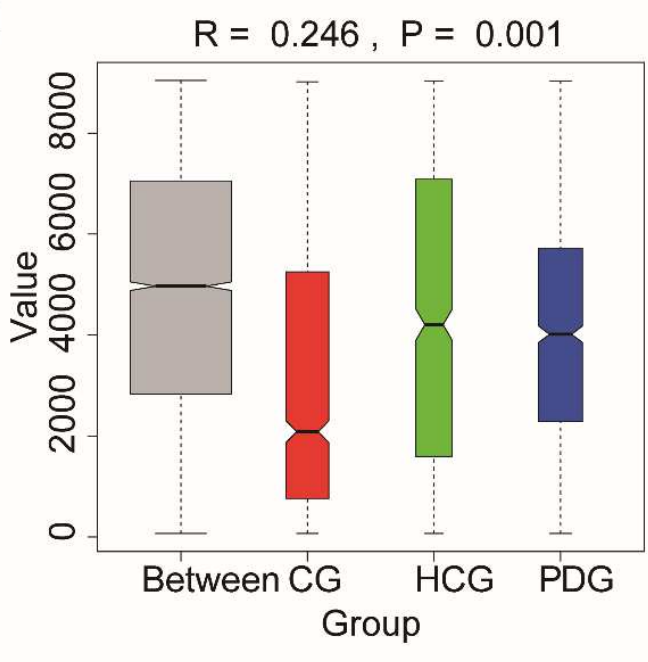

658

659

660

661

662

663

Fig.4 Oropharynx microbial alterations in diversity among HCG, PDG and CG.

a Little changes in Observed index among HCG, PDG and CG (NS: $P>0.05$ ).

b Little changes in Shannon index among HCG, PDG and CG (NS: $P>0.05)$. c

Principal coordinate analysis (PCoA) of Bray-Curtis distance showed obviously changes in $\beta$-diversity among HCG, PDG and CG. The colors represent three different groups. PCoA1 and PCoA2 represent the top two principal coordinates that captured most of the diversity. The fraction of diversity captured by the 
a
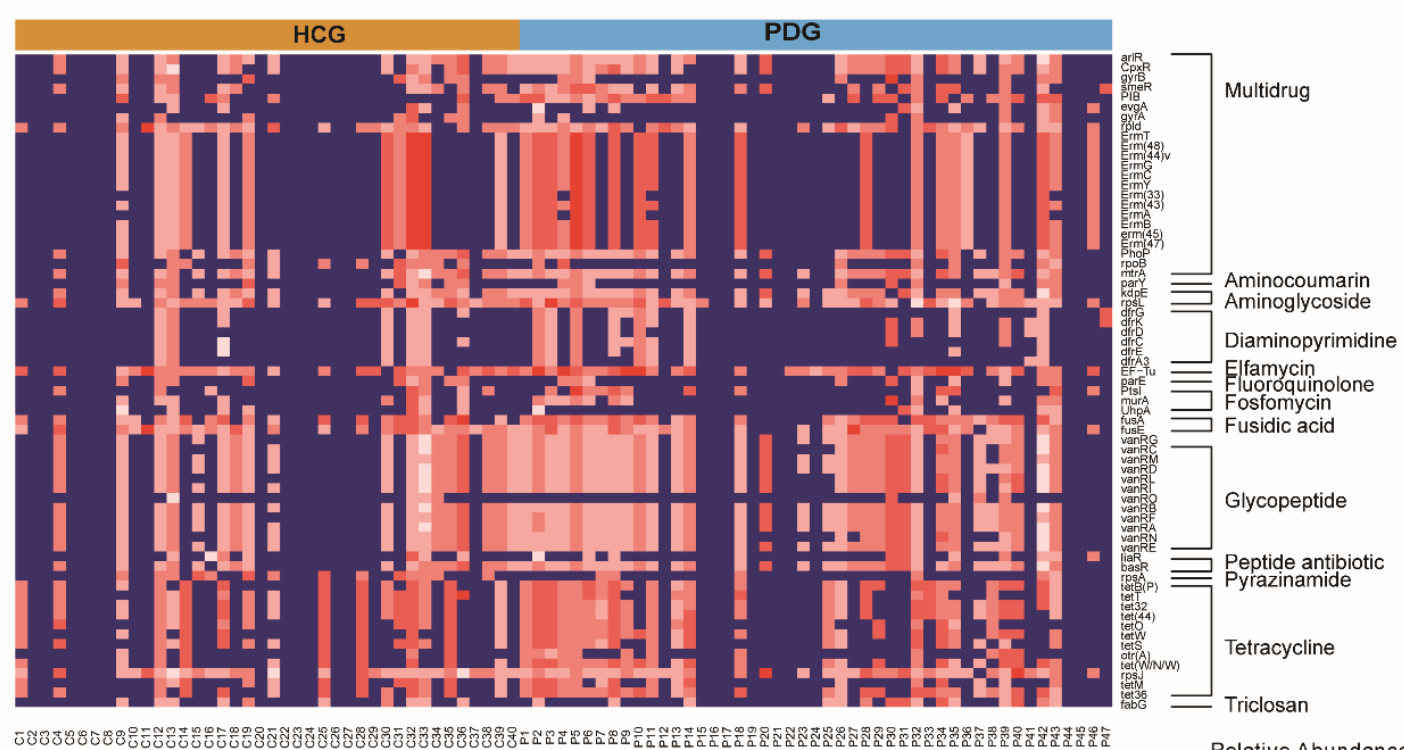

b

Relative Abundance
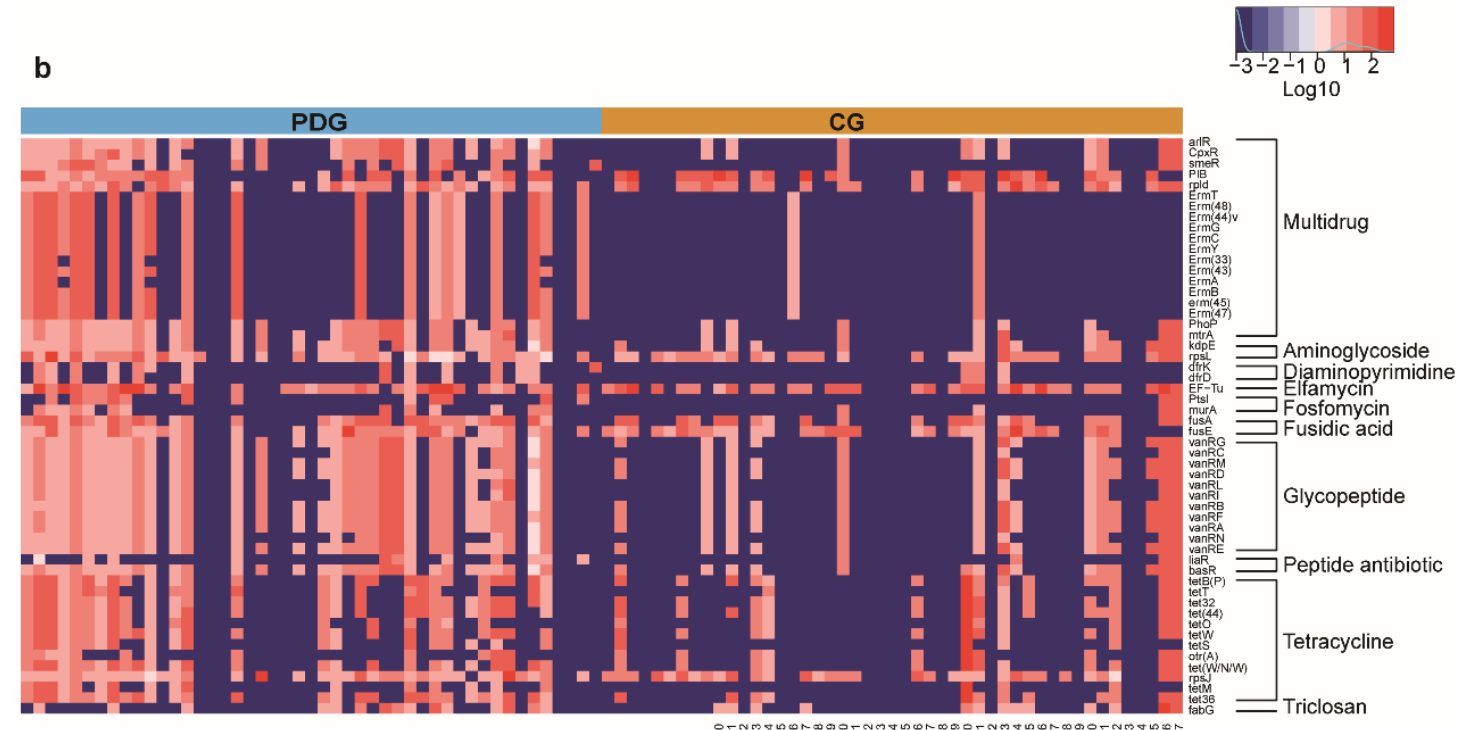

672

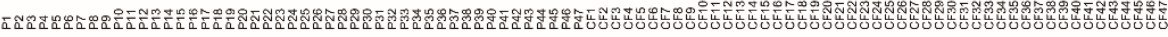


675 of microbial antibiotic resistance genes expression between HCG and PDG. $\mathbf{b}$

677 expression between PDG and CG. The relative abundance changes from low

678 to high is showed by color changes from blue to red. The log10 value was used

679 to show the abundance of antibiotic resistance gene.

a

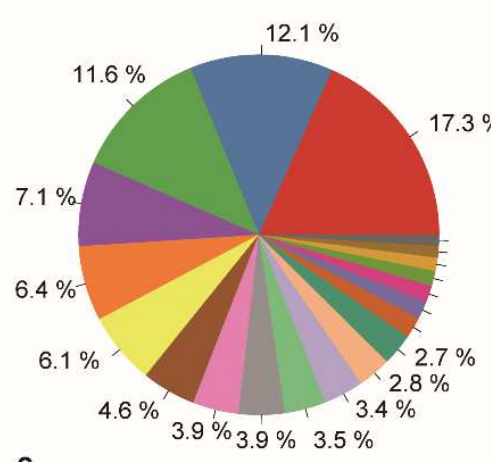

c

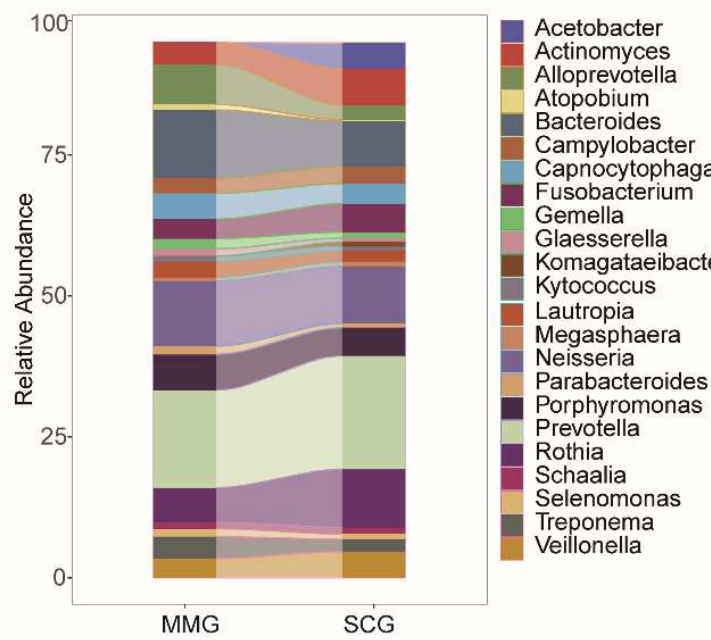

e

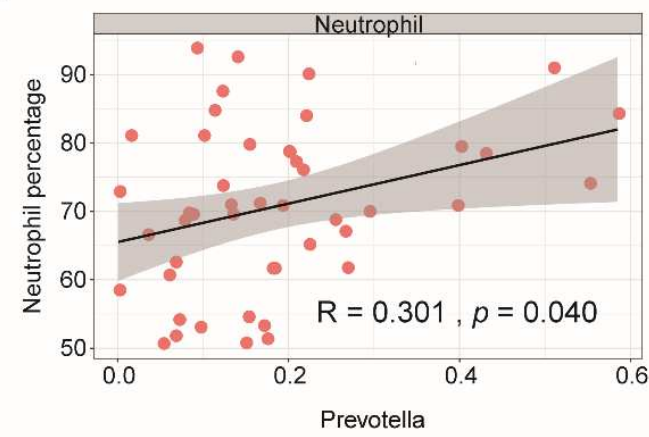

b

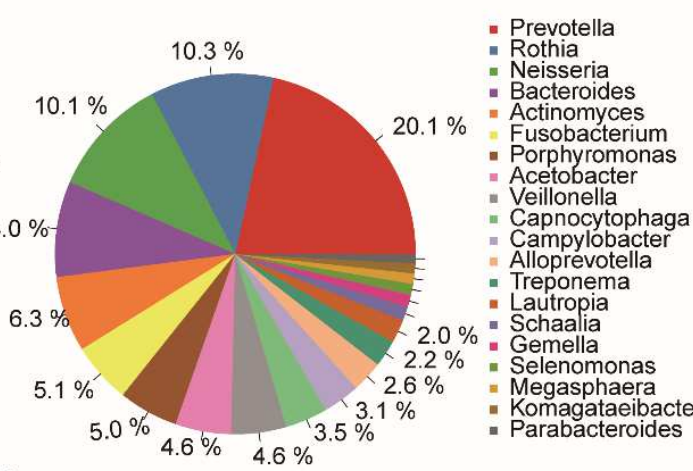

d

Prevotella

- Neisseria

Porphyromona

Rothia

- Capnocytophaga

Treponema

Fusobacterium $8.0 \%$

- Veillonella

- Lautropia

- Gemella

- Parabacteroides

- Glenomonas

Schaalia

- Atopobium

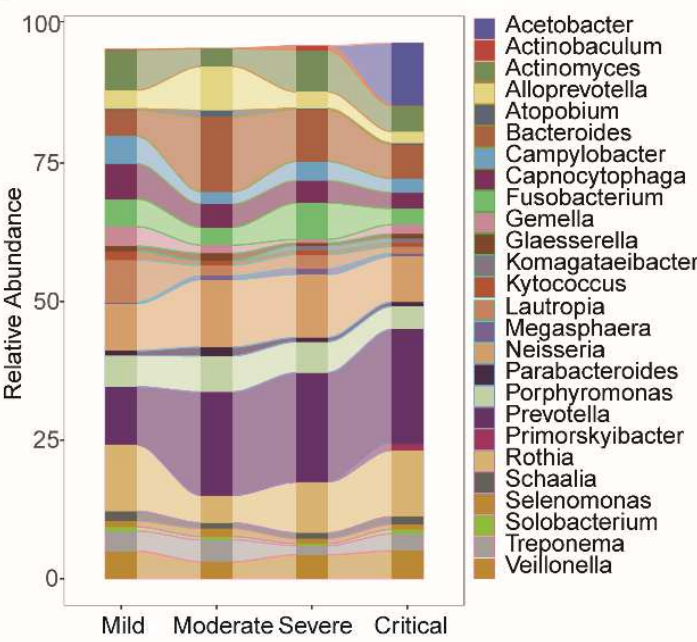


Fig.6 Alterations of oropharyngeal bacteria reflected disease severity in

682 patients with COVID-19. a, b Average compositions of relative abundance of 683 the top20 bacterial communities of MMG and SCG groups at the genus level. c 684 Alterations of top20 bacterial communities among the MMG and SCG groups 685 at the genus level. d Alterations of top20 bacterial communities among the Mild, 686 Moderate, Severe and Critical groups at the genus level. Especially, the 687 alteration of Prevotella gradually increased along with the symptom aggravated 688 in patients with COVID-19. e Positive correlation between Prevotella relative 689 abundance (x-axis) and the elevation of Neutrophil percentage (y-axis) 690 (Spearman correlation analysis: $\mathrm{R}=0.301, p=0.040$ ), Linear regression lines 691 are shown in each scatter plot in black, and shaded regions represent $95 \%$ 692 confidence intervals. 


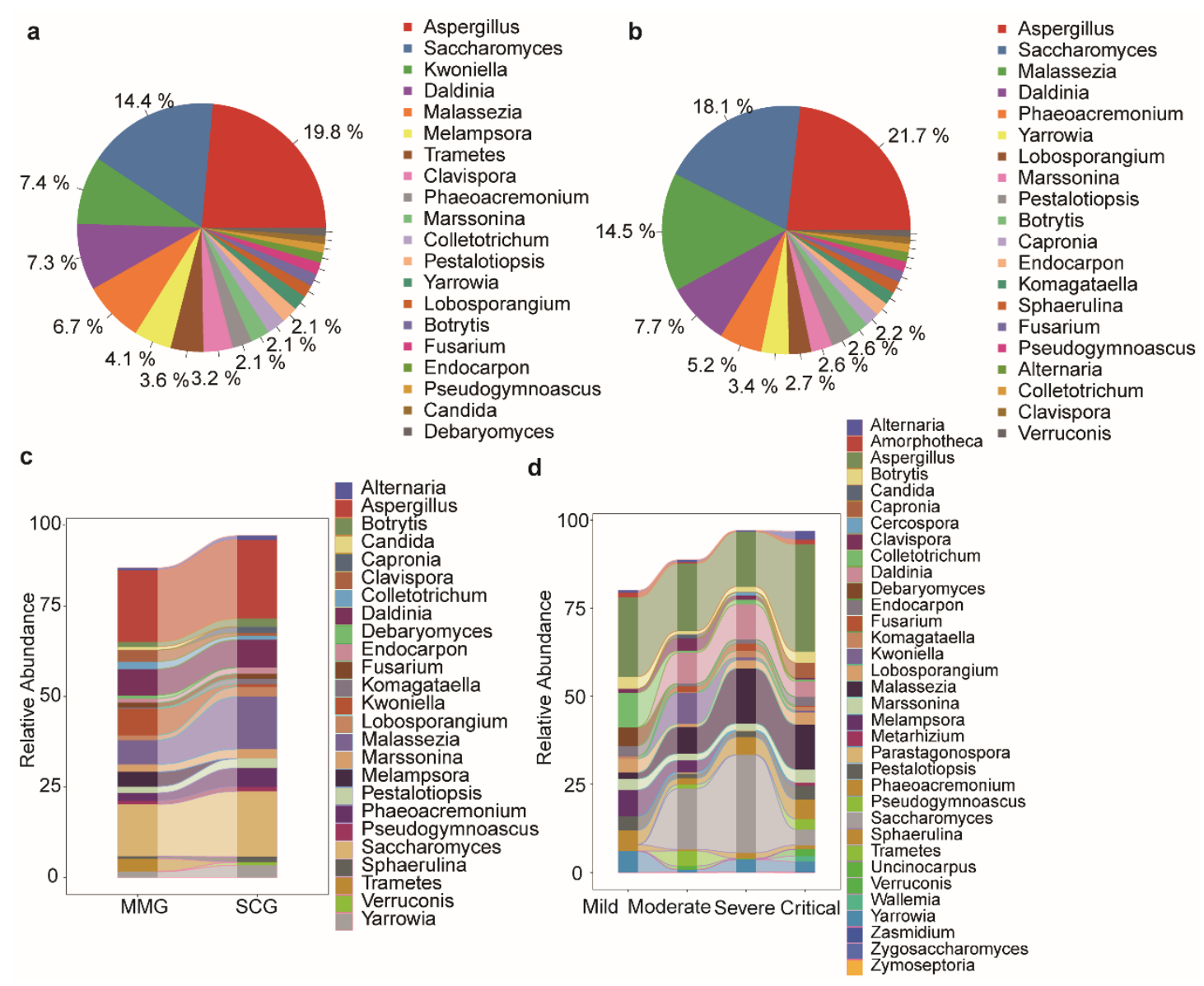

Fig.7 Alterations of oropharyngeal fungi reflected disease severity in patients level. d Alterations of top20 fungal communities among the Mild, Moderate, remarkably in critical Patients with COVID-19. 


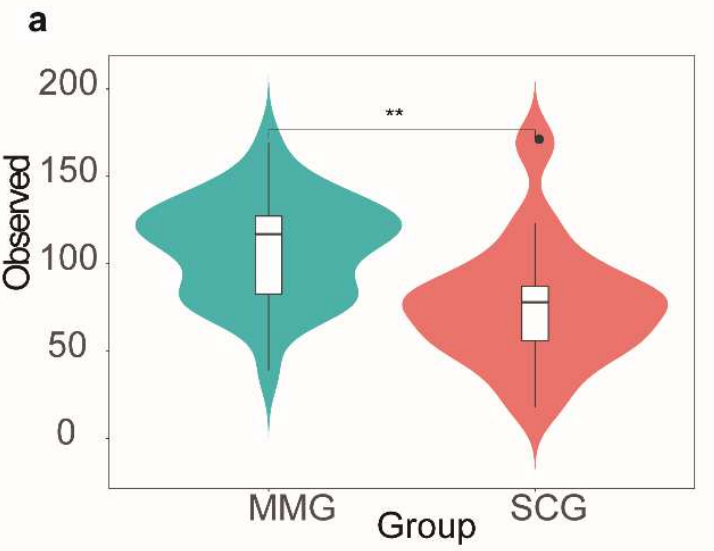

C

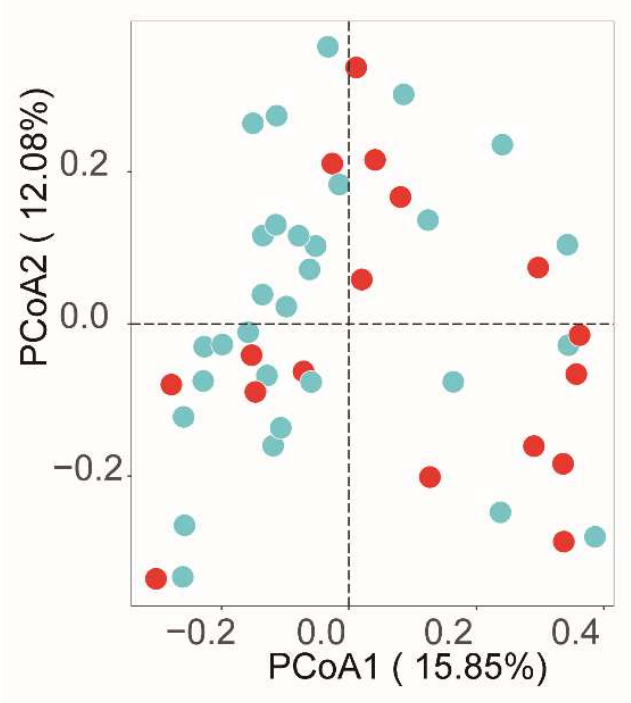

b

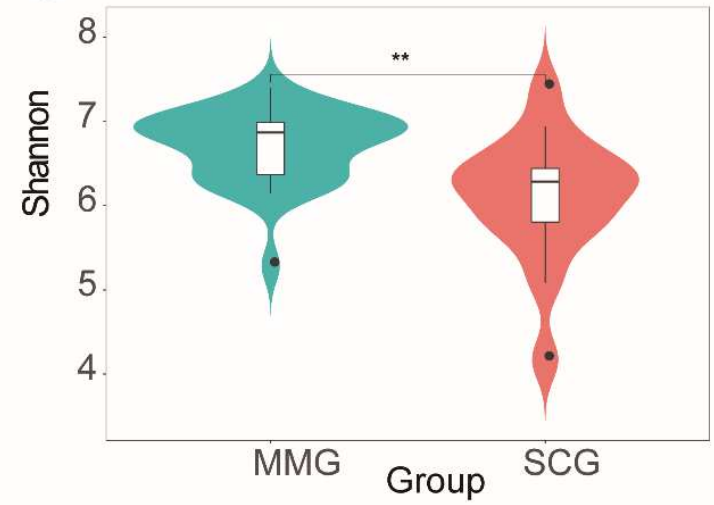

d
701

702

703

704

705

706

707

708

709

710

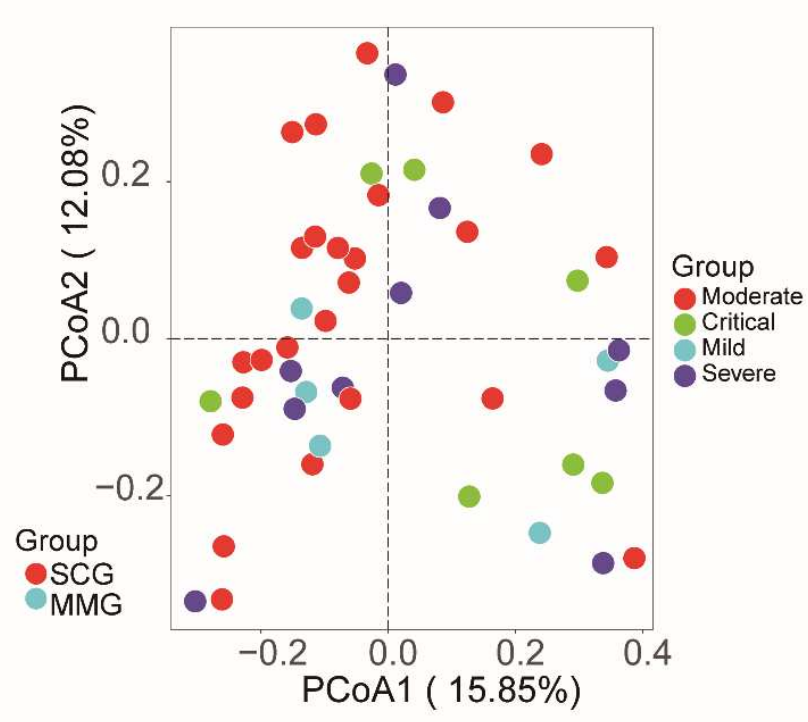

Fig.8 Decreased oropharyngeal microbiome in diversity appeared to be associated with COVID-19 severity. a Obviously decreased oropharyngeal microbiome in Observed index between MMG and SCG $(* *$ : $P<0.001)$. b Obviously decreased oropharyngeal microbiome in Shannon index between MMG and SCG $\left({ }^{* *}: P<0.001\right)$. c Principal coordinate analysis (PCoA) of BrayCurtis distance showed obviously changes in $\beta$-diversity among MMG and SCG groups. d Principal coordinate analysis (PCoA) of Bray-Curtis distance showed obviously changes in $\beta$-diversity among Mild, Moderate, Severe and Critical groups. The colors represent different groups. PCoA1 and PCoA2 represent 
712 fraction of diversity captured by the coordinate is valued as a percentage.

a
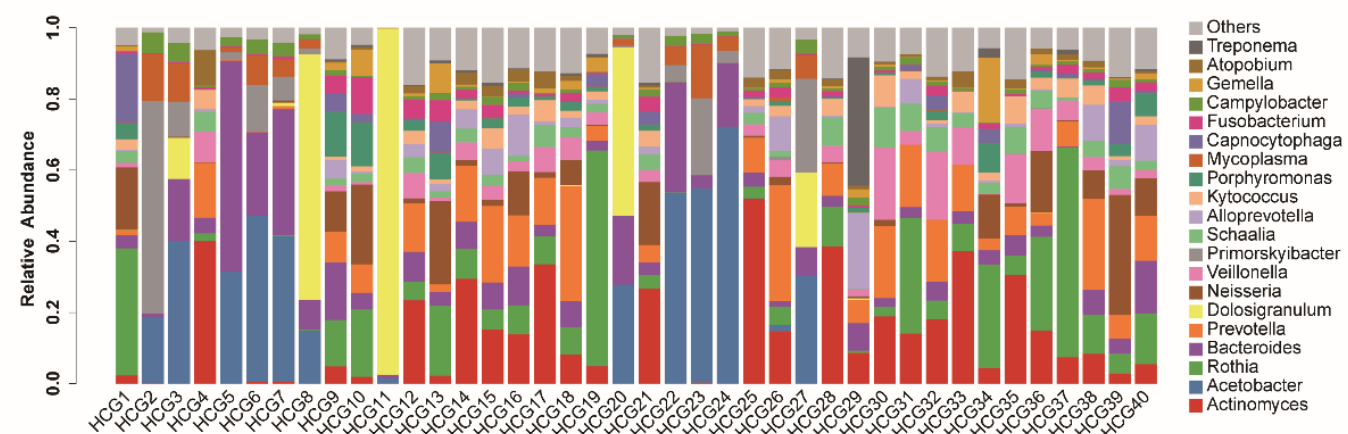

b
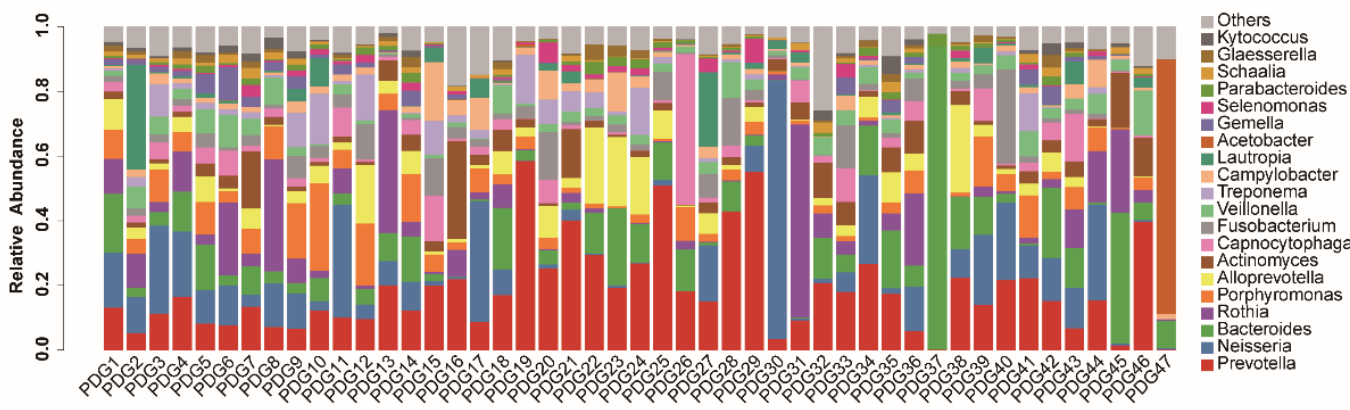

C
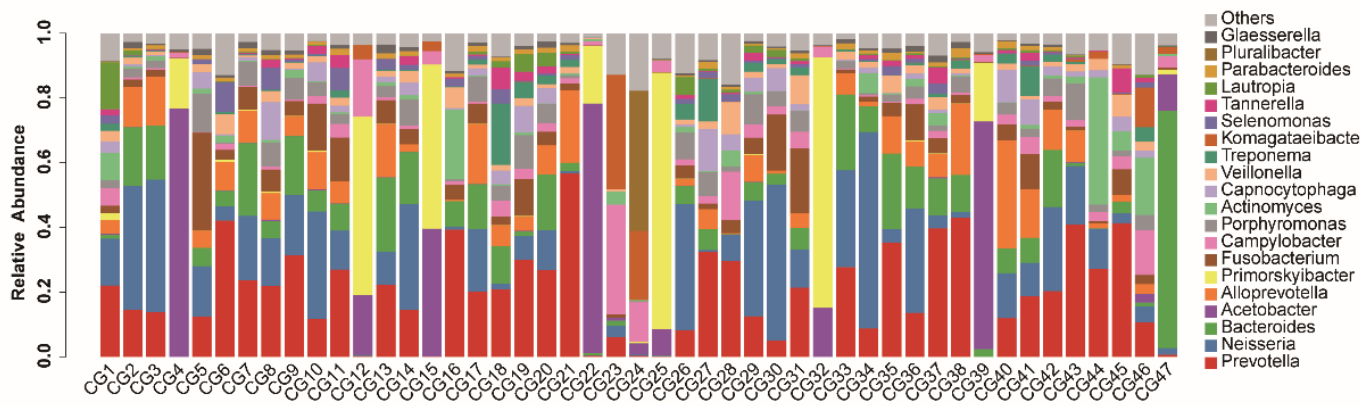

Suppl.Fig.1 Oropharyngeal bacterial characterization of every subjects among

HCG, PDG and CG. Average compositions of relative abundance of the top20

bacterial communities for each group at the genus level, a for HCG, $\mathbf{b}$ for PDG and $\mathbf{c}$ for CG. shared bacterial landscape on genus level among HCG, PDG and CG group. 

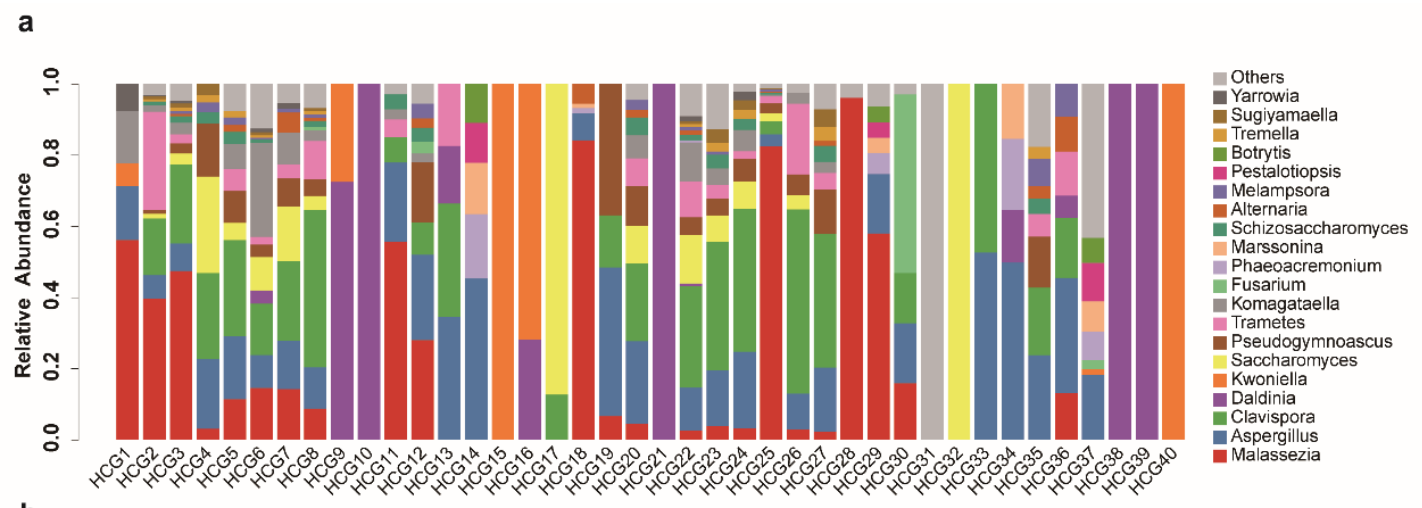

b
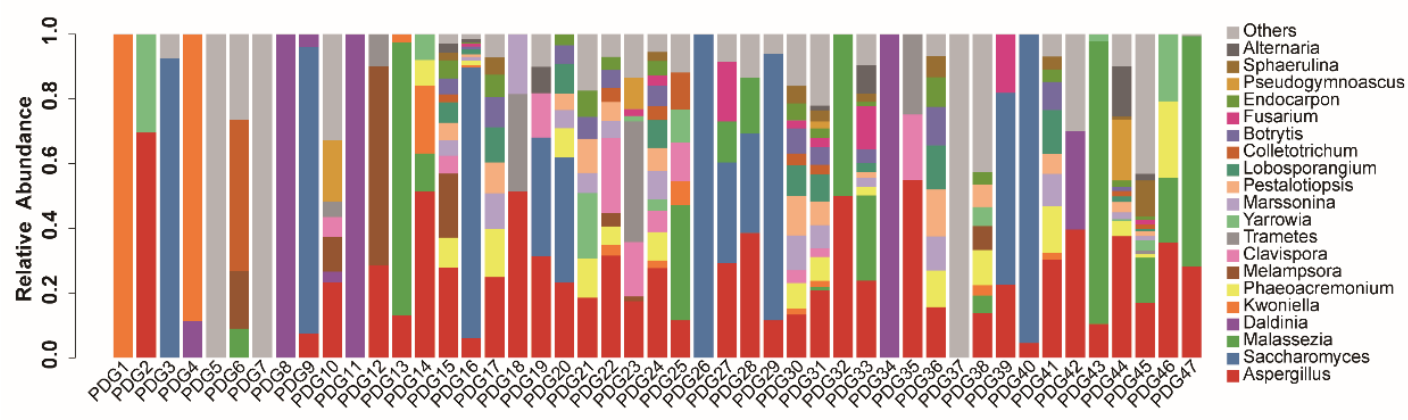

c
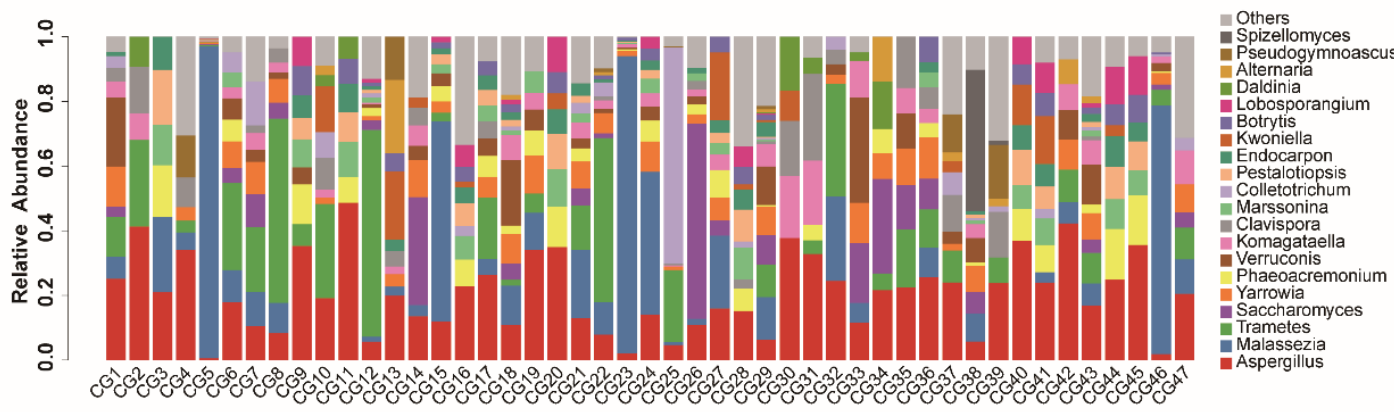

Suppl.Fig.1 Oropharyngeal fungal characterization of every subjects among and $\mathbf{c}$ for $\mathrm{CG}$. shared bacterial landscape on genus level among HCG, PDG and CG group. 


\section{Figures}

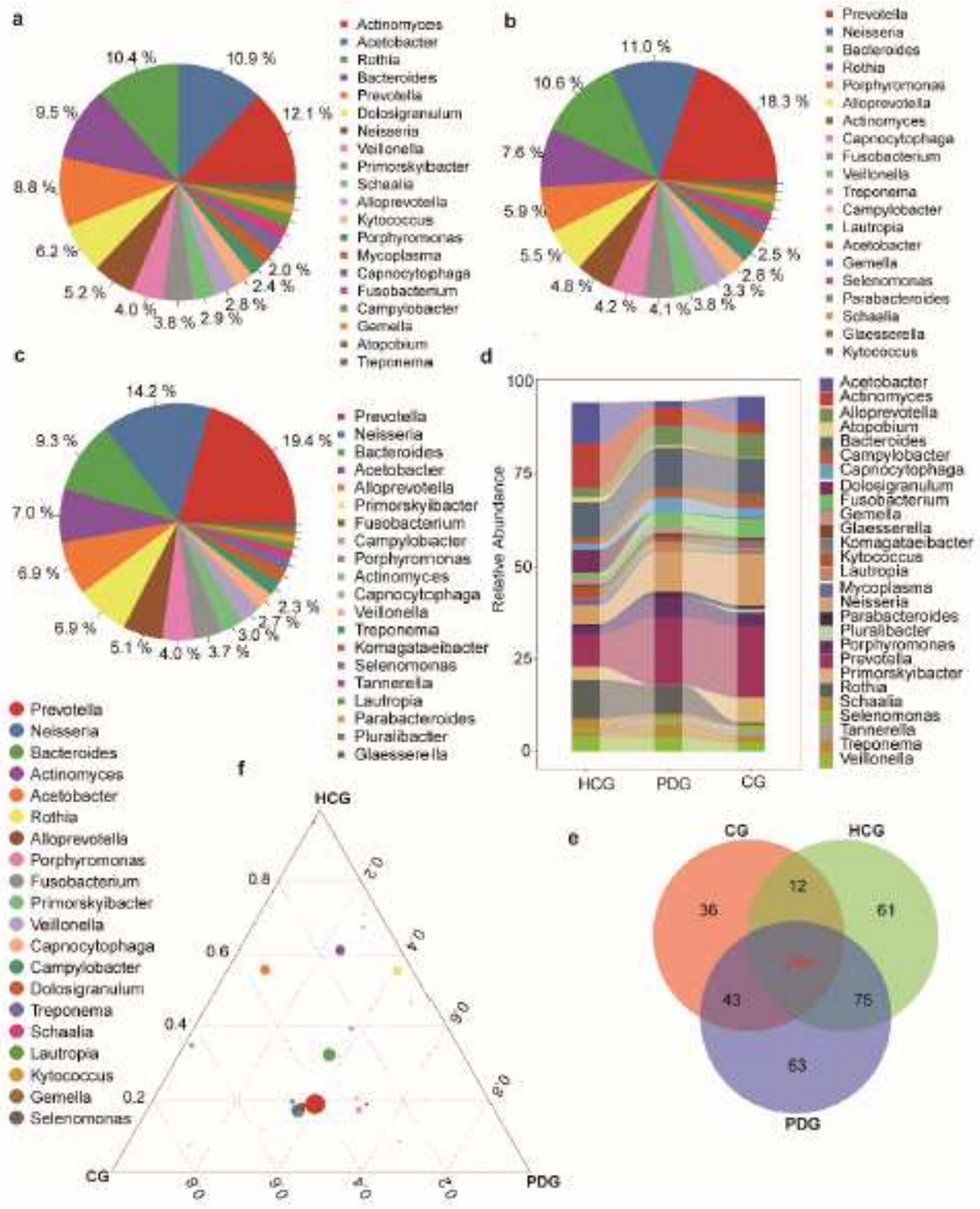

\section{Figure 1}

Oropharyngeal bacterial characterization and alterations among HCG, PDG and CG. Average compositions of relative abundance of the top20 bacterial communities for each group at the genus level, a for HCG, $b$ for PDG and c for CG. $d$ Alterations of top20 bacterial communities among the three groups at the genus level, Especially, Prevotella is significantly high er in PDG than that in HCG (Wilcoxon rank

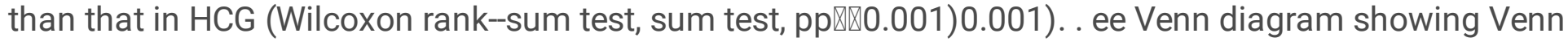
diagram showing shared and unique genus of the three groups. shared and unique genus of the three groups. ff Ternary plots depicting the 244 Ternary plots depicting the 244 shared bacterial landscape on genus level among HCG, PDG and CG group. shared bacterial landscape on genus level among HCG, PDG and CG group. The sum of the proporThe sum of the proportion for one specific bacteria in the three groups was set tion for one specific bacteria in the three groups was set as 1 , and the proportion of one 
specific bacteria in each group is equal to its as 1 , and the proportion of one specific bacteria in each group is equal to its corresponding relative abundance divided by the relative abundance sum of corresponding relative abundance divided by the relative abundance sum of this bacteria in the three groups. The sizethis bacteria in the three groups. The size of the circles represents the relative of the circles represents the relative abundance of the genus.abundance of the genus.

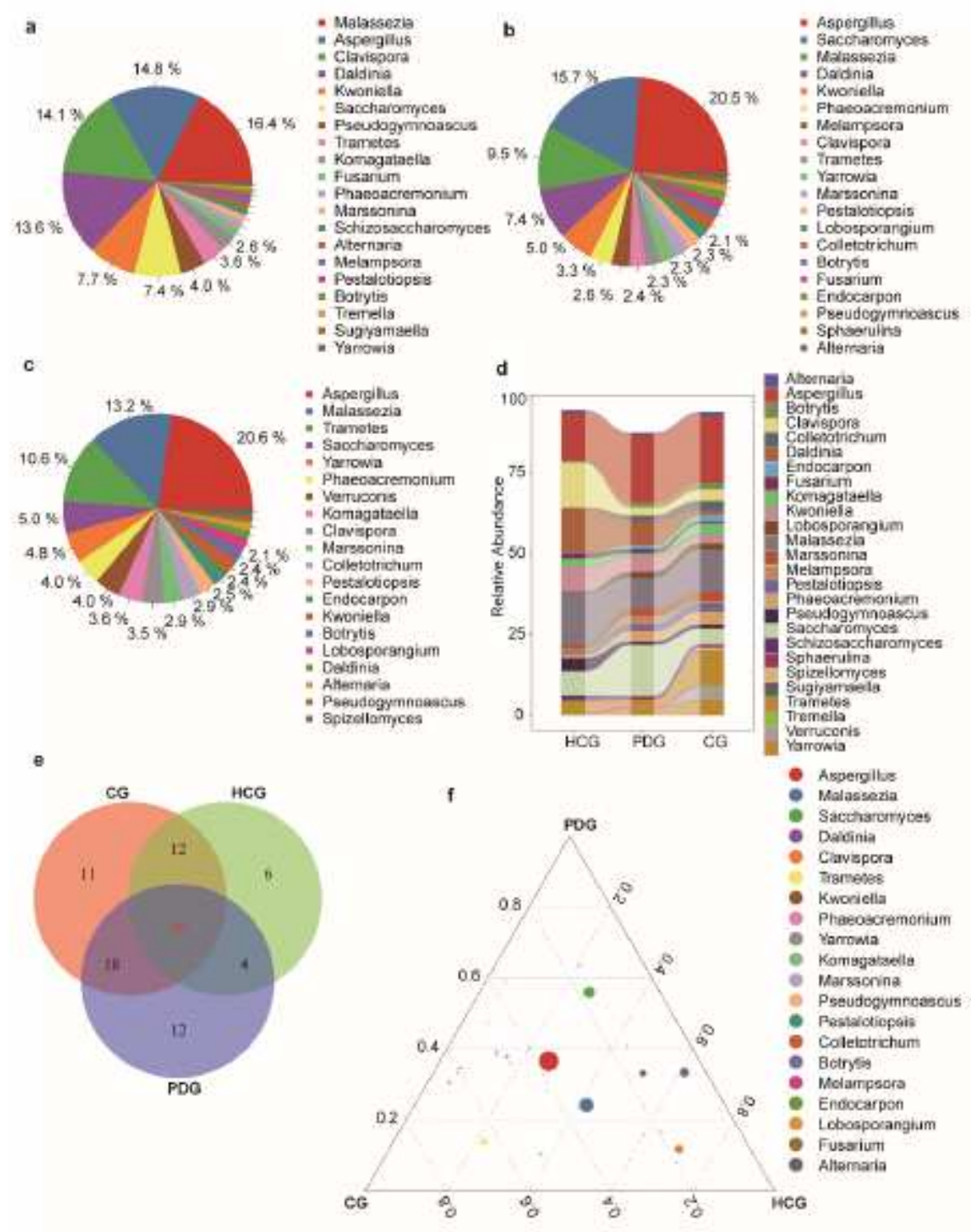

Figure 2

Oropharyngeal fungal characterization and alterations among HCG, PDG and CG. Average compositions of relative abundance of the top20 fungal communities for each group at the genus level, a for HCG, $b$ for PDG and $\mathrm{c}$ for CG. $d$ Alterations of top20 fungal communities among the three groups at the genus level. e Venn diagram showing shared and unique genus of the three groups. groups. ff Ternary plots depiTernary plots depicting the 244 shared cting the 244 shared fungalfungal landscape on genus landscape on genus level among HCG, PDG and CG group. The sum of the proportion for one level among HCG, PDG and CG group. The sum of the proportion for one specific bacteria in the three groups was set as 1 , and the proportion of one specific bacteria in the three groups was set as 1 , and the 
proportion of one specific fungi in each group is equal to its corresponding relspecific fungi in each group is equal to its corresponding relative abundance ative abundance divided by the relative abundance sum of this fungi in the three groups. The divided by the relative abundance sum of this fungi in the three groups. The size of the circles represents the relative abundance of the genus.size of the circles represents the relative abundance of the genus.
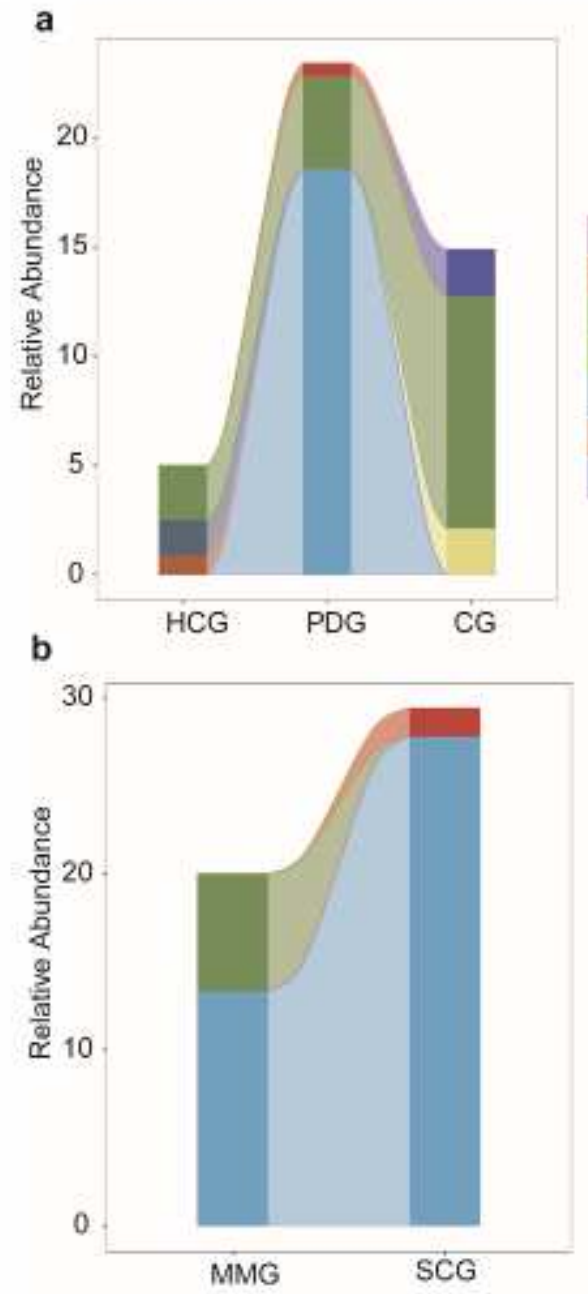

Human alphaherpesvirus 1 Human betaherpesvirus 7 Human gammaherpesvirus 4 Porcine type $\mathrm{C}$ oncovirus Rhinovirus A Rhinovirus $\mathrm{C}$ SARS-CoV-2 c

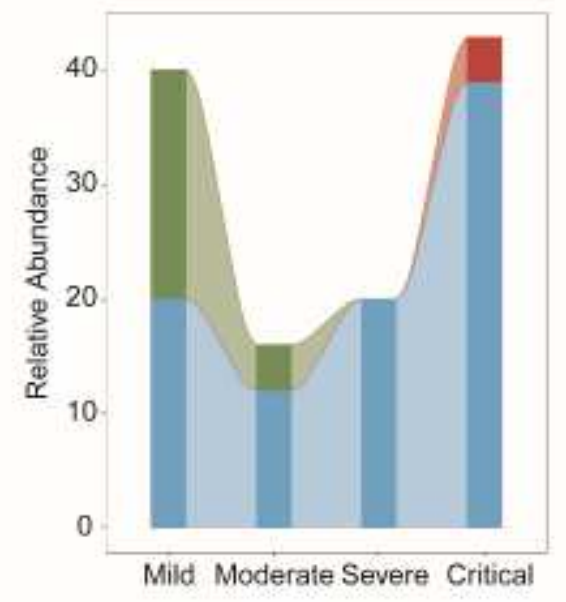

d

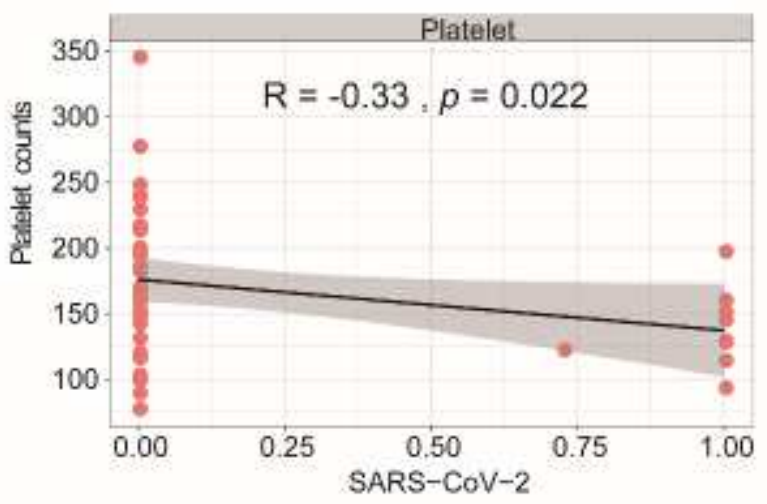

\section{Figure 3}

Oropharyngeal SARS CoV 2 viral load was positively correlated with the severity of COVID 19. a The relative abundance changes of 7 oropharyngeal viral species (including SARS-COR-2) among HCG, PDG and CG group, b among MMG and SCG group, $c$ among Mild, Moderate, Severe and Critical group. $d$ Negative correlation between SARS-CoV-2 viral load (x-axis) and platelet counts (y-axis) (Spearman correlation analysis: $R=-0.330, P=0.022)$, Linear regression lines are shown in each scatter plot in black, and shaded regions represent $95 \%$ confidence intervals. 
a

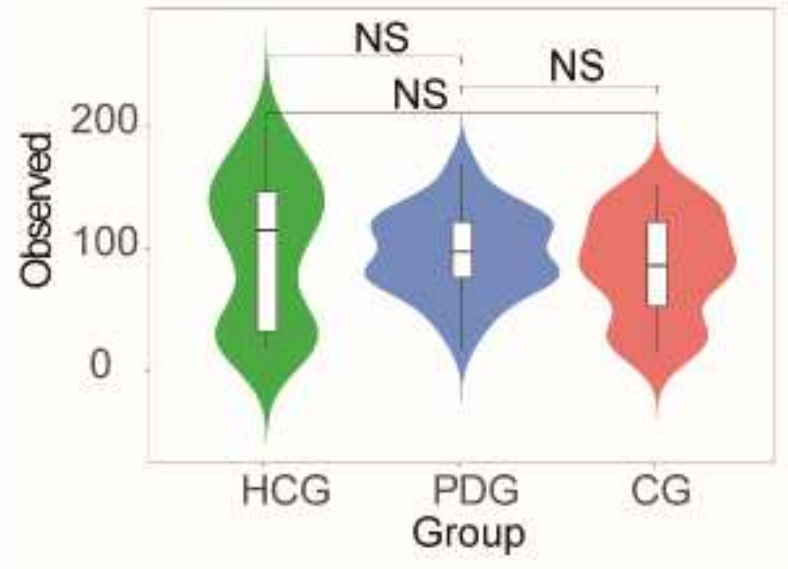

C

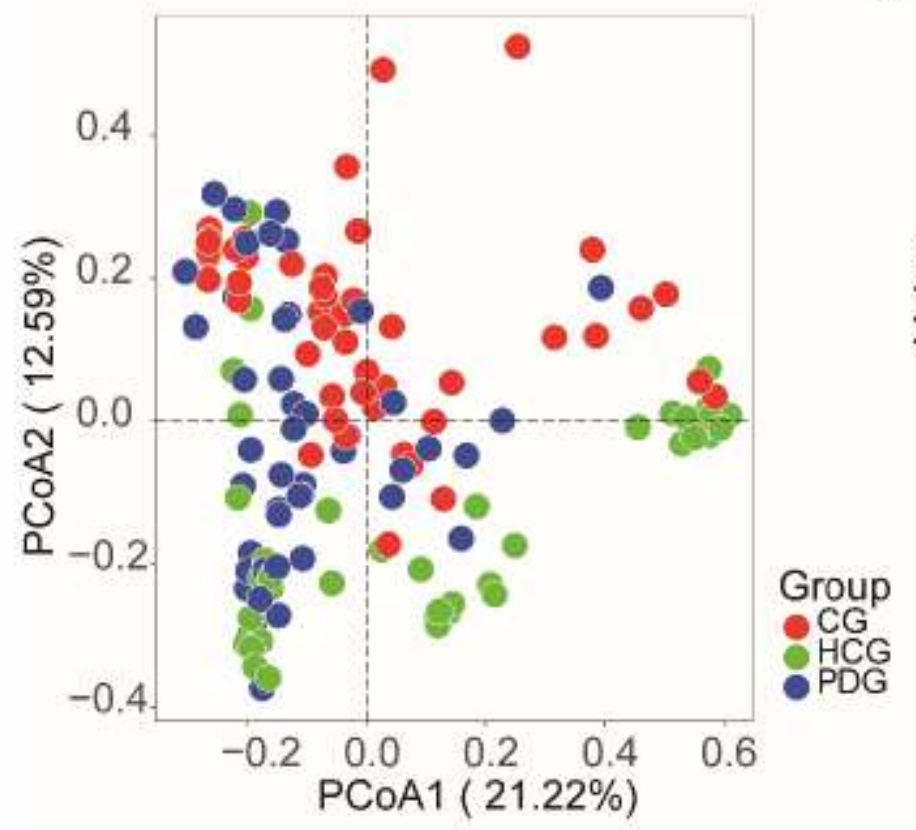

b

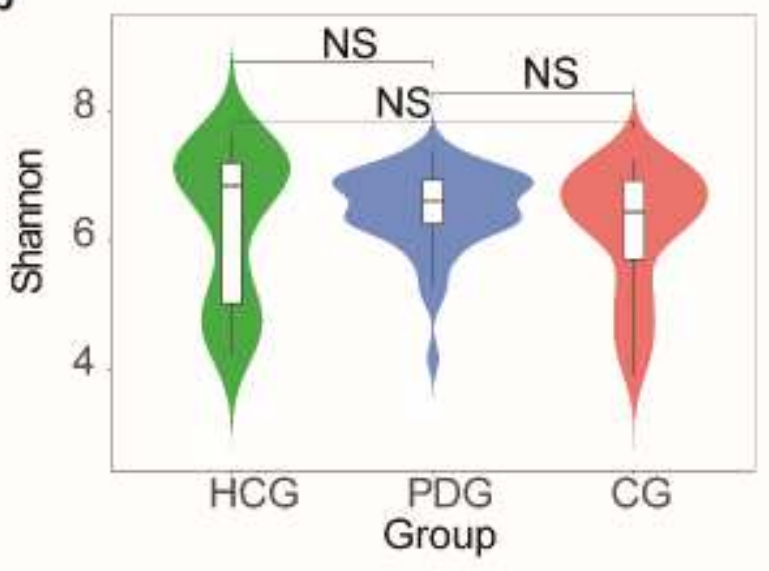

d

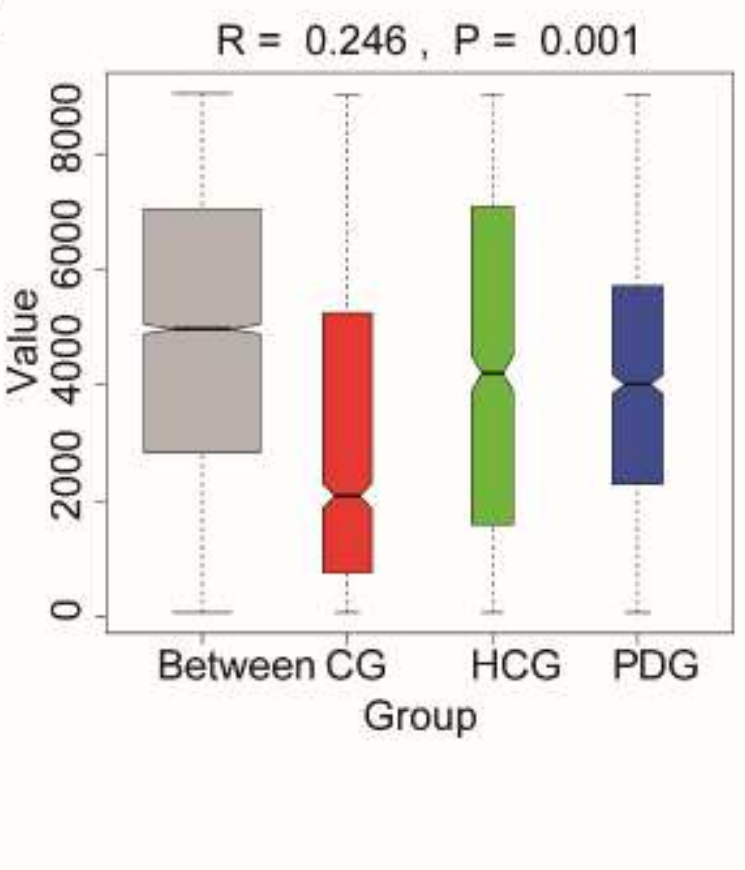

\section{Figure 4}

Oropharynx microbial alterations in diversity among HCG, PDG and CG. a Little changes in Observed index among HCG, PDG and CG (NS: P 0.05). b Little changes in Shannon index among HCG, PDG and CG (NS: P 0.05). c Principal coordinate analy sis (PCoA) of Bray Curtis distance showed obviously changes in $\beta$ diversity among HCG, PDG and CG. The colors represent three different groups. PCoA1 and PCOA2 represent the top two principal coordinates that captured most of the diversity. The fraction of diversity captured by the coordinate is valued as a percentage. coordinate is valued as a percentage. dd. The analysis of similarities (ANOSIM). The analysis of similarities (ANOSIM) based on Braybased on Bray--Curtis distance shows that $R>0$ and Curtis distance shows that $R>0$ and $P P<0.05$, indicating that $<0.05$, indicating that the interthe inter-group difference among HCG, PDG and CG was greater than the intragroup difference among HCG, PDG and CG was greater than the intra-group difference in each group. The R means the statistical value of ANOSIM, group difference in each group. The $\mathrm{R}$ means the statistical value of ANOSIM, yy--axis value means Brayaxis value means Bray--Curtis rank, and 999 was 
used for the permutations.Curtis rank, and 999 was used for the permutations. NSNS means no significant.means no significant.

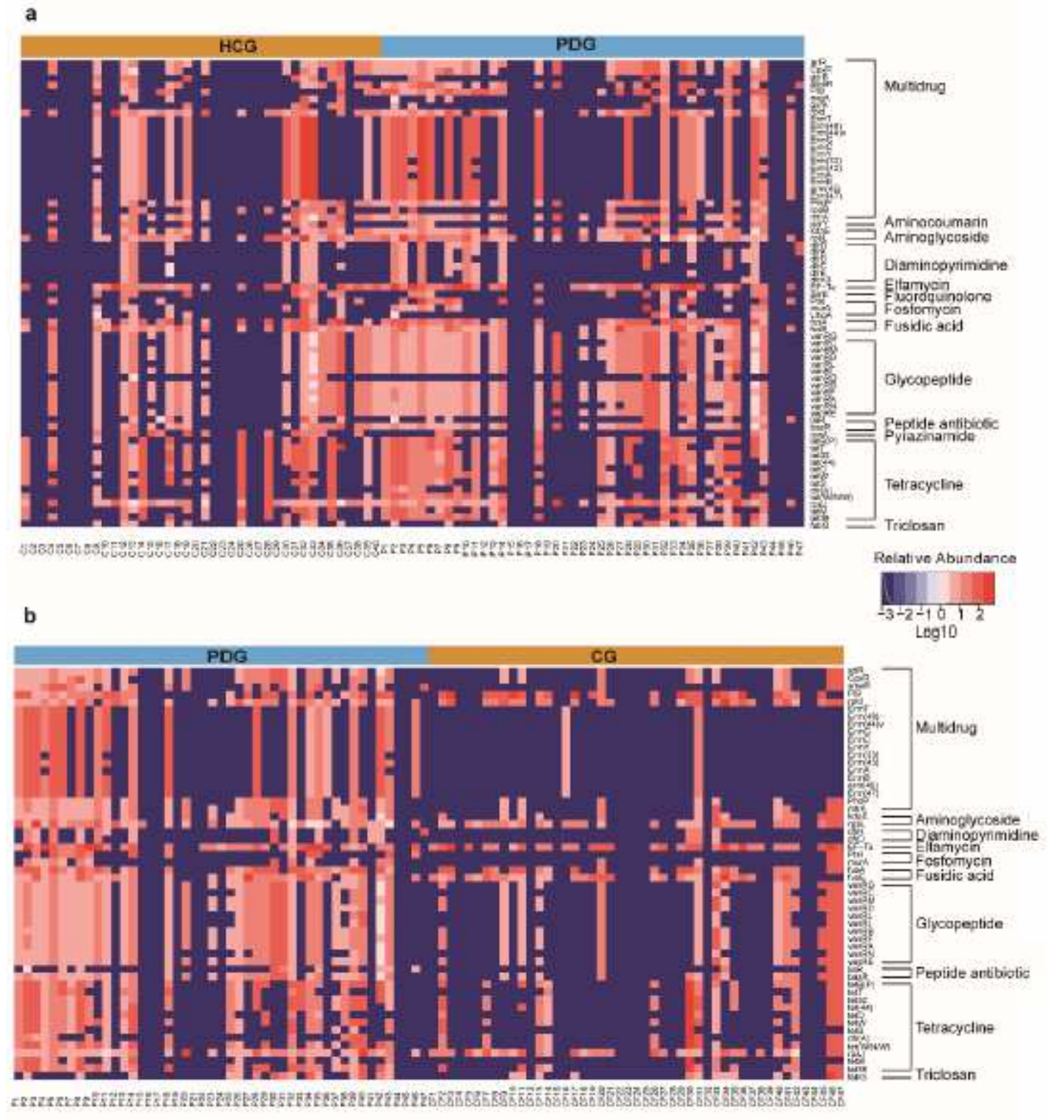

\section{Figure 5}

Obviously changes of oropharynx microbial antibiotic resistance genes expression among HCG, PDG and CG. a expression among HCG, PDG and CG. a Heatmap for the relative abundances Heatmap for the relative abundances of microbial antibiotic resistance genes expression between HCG and PDG. of microbial antibiotic resistance genes expression between HCG and PDG. bb Heatmap Heatmap for the relative abundances of microbial antibiotic resistance genes for the relative abundances of microbial antibiotic resistance genes expression between PDG and CG. The relative abundance changes from low expression between PDG and CG. The relative abundance changes from low to high is showed by color changes from blue to red. The log10 value was used to high is showed by color changes from blue to red. The log10 value was used to show the abundance ofto show the abundance of antibiotic reantibiotic resistance genesistance gene.. 
a
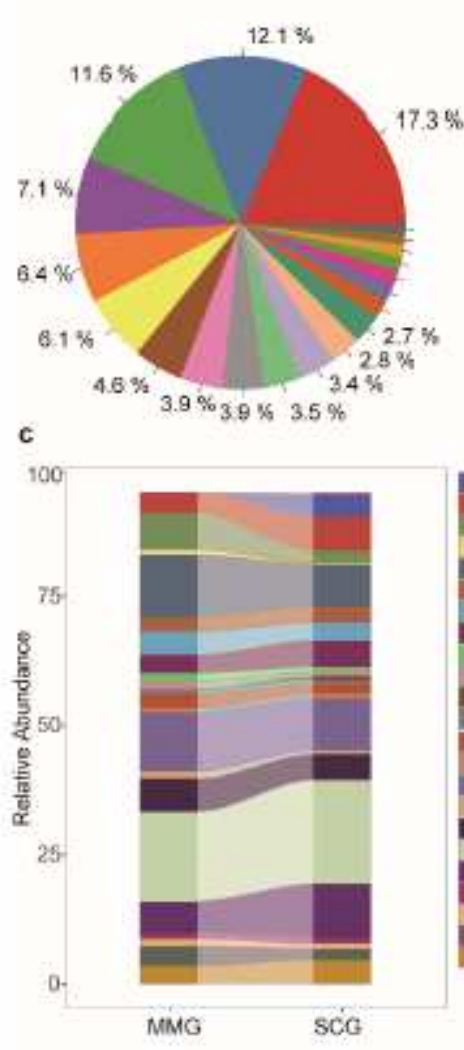

e

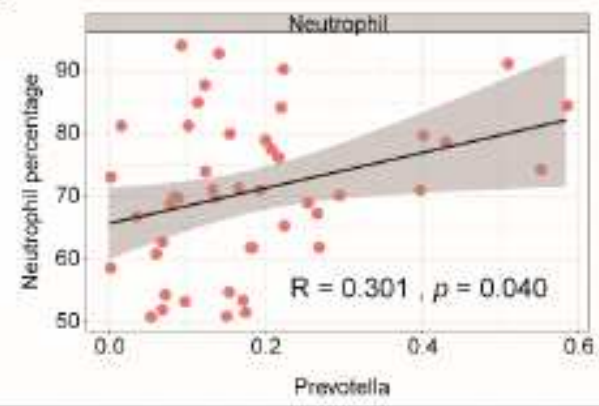

b

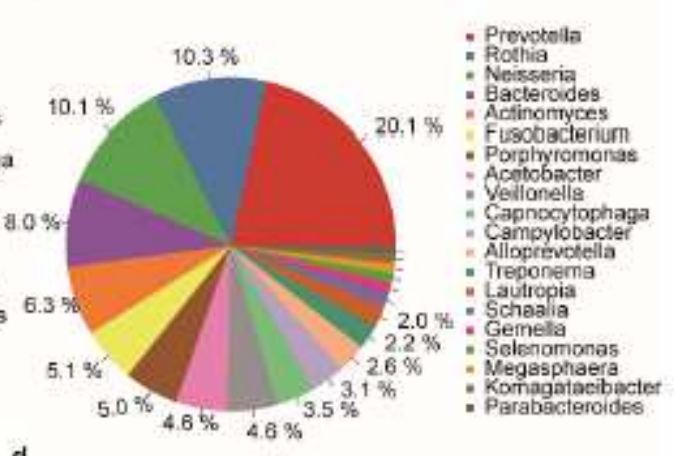

$$
\text { d }
$$

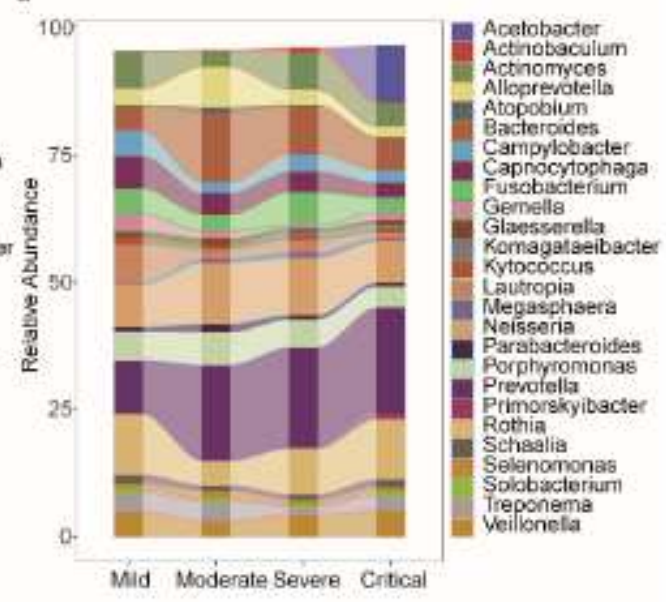

Figure 6

Alterations of oropharyngeal bacteria reflected disease severity in patients with COVID 19. a, b Average compositions of relative abundance of the top20 bacterial communities of MMG and SCG groups at the genus level. c Alterations of top20 bacterial communities among the MMG and SCG groups at the genus level. $d$ Alterations of top20 bacterial communities among the Mild, Moderate, Severe and Critical groups at the genus level. Especially, the alteration of Prevotella gradually increased along with the symptom aggravated in patients with COVID 19. e Positive correlation between Prevotella relative abundance ( $x$ axis) and the elevation of Neutrophil percentage ( $y$-axis) (Spearman correlation analysis: $R=0.301, p=$ 0.040), Linear regression lines are shown in each scatter plot in black, and shaded regions represent $95 \%$ confidence intervals 


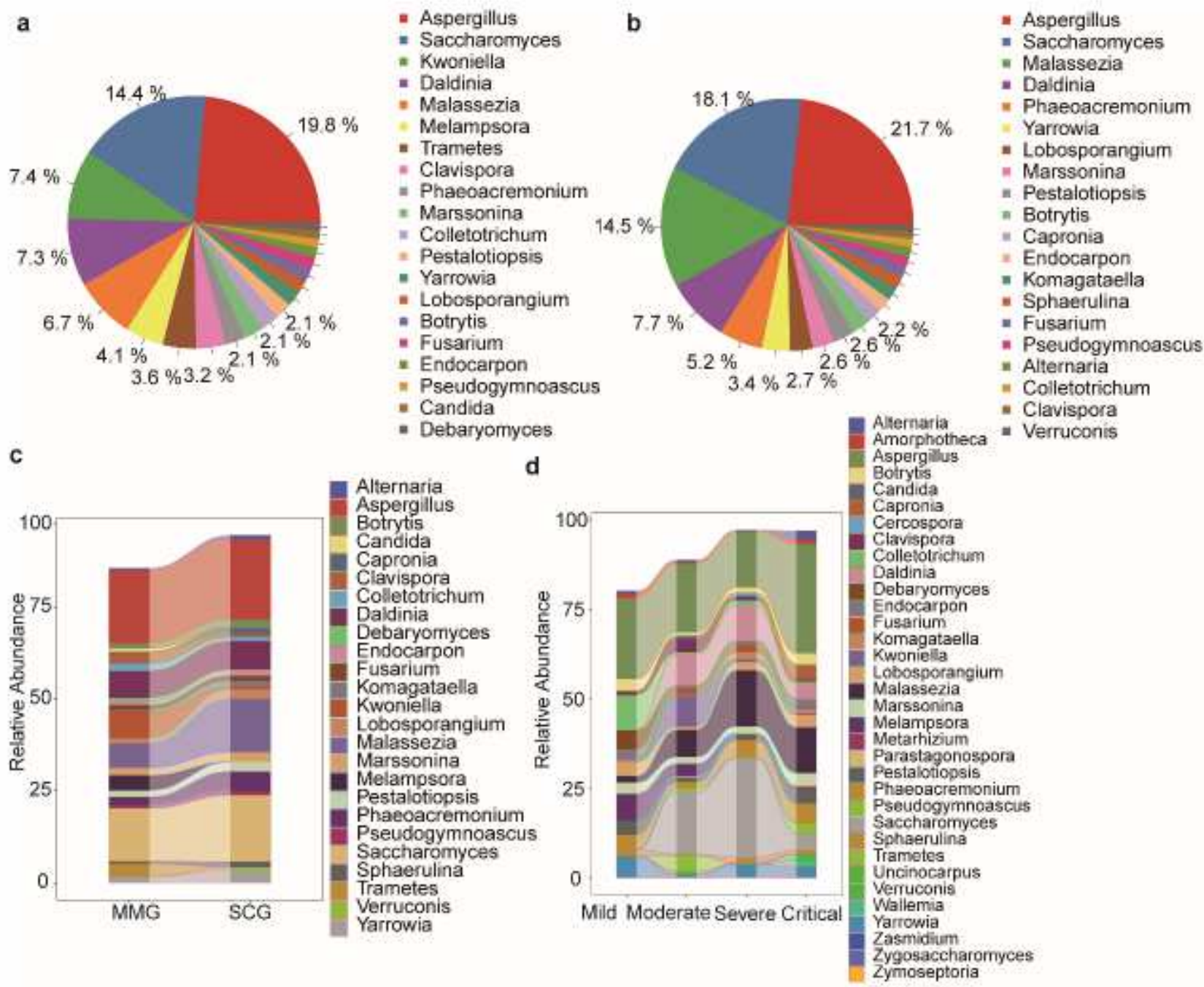

Figure 7

Alterations of oropharyngeal fungi reflected disease severity in patients with COVID 19. a, b Average compositions and relativ e abundance of the top20 fungal communities of MMG and SCG groups at the genus level. c Alterations of top20 fungal communities among the MMG and SCG groups at the genus level. d Alterations of top20 fungal communities among the Mild, Moderate, Severe and Critical groups at the genus level. Especially Aspergillus increased remarkably in critical Patients with COVID 19. 
a

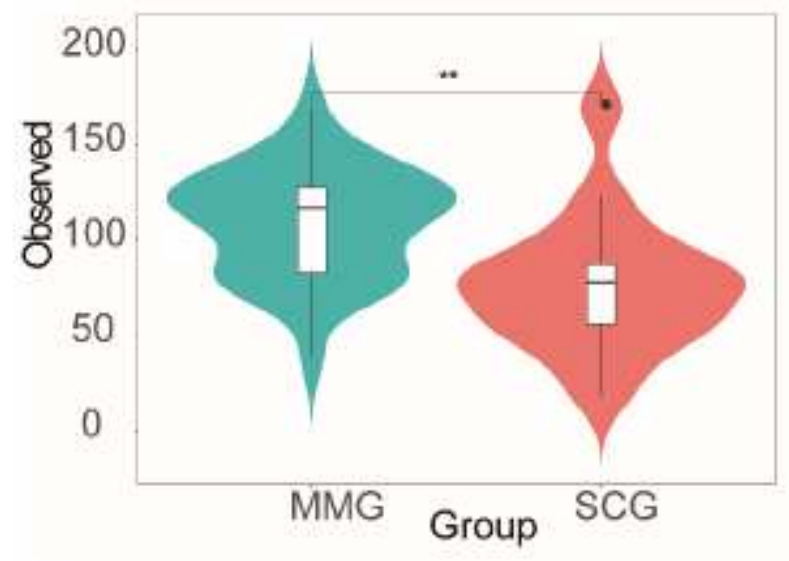

C

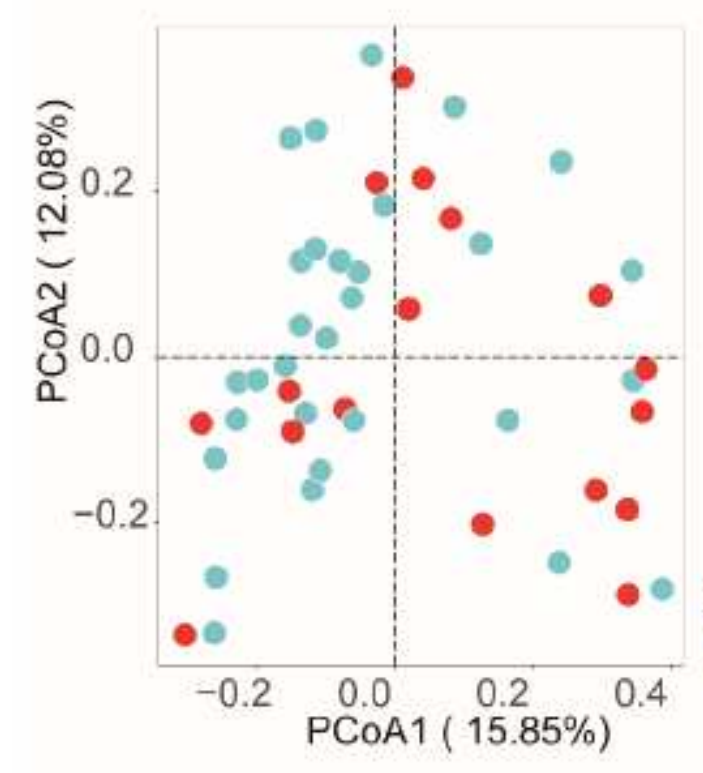

b

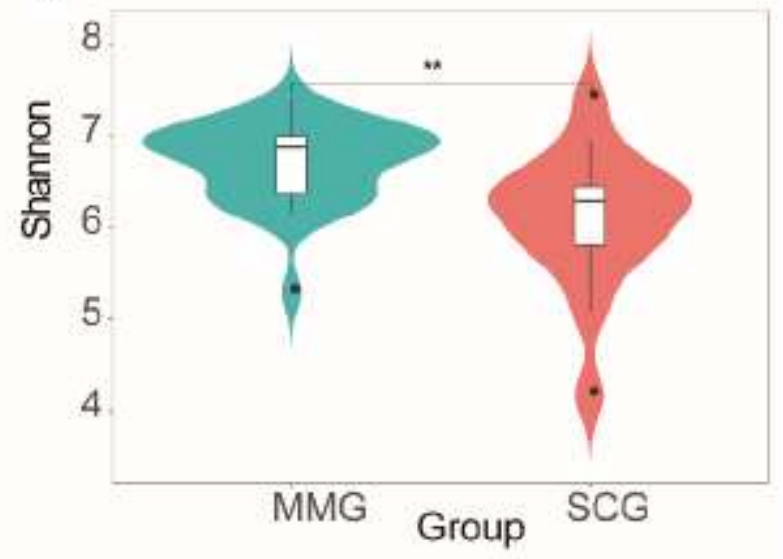

d

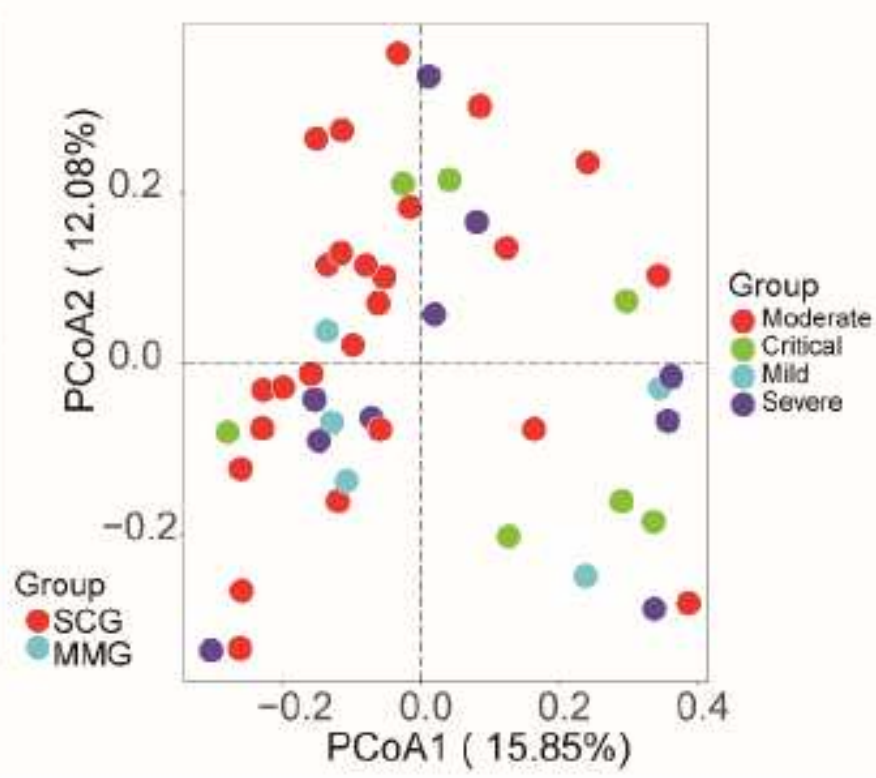

\section{Figure 8}

Decreased oropharyngeal microbiome in diversity appeared to be associated with COVID 19 severity. a Obviously decreased oropharyngeal microbiome in Observed index between MMG and SCG (**: P 0.001). b Obviously decreased oropharyngeal microbiome in Shannon index between MMG and SCG (**: P 0.001). c Principal coordinate analysis (PCoA) of Bray Curtis distance showed obviously changes in $\beta$ diversity among MMG and SCG groups. d Principal coordinate analysis (PCOA) of Bray Curtis distance showed obviously changes in $\beta$ diversity among Mild, Moderate, Severe and Critical groups. The colors represent different groups. PCOA1 and PCOA 2 represent the top two principal coordinates that captured most of the diversity. The the top two principal coordinates that captured most of the diversity. The fraction of diversity captured by the coordinate is valued as a percentage.fraction of diversity captured by the coordinate is valued as a percentage.

\section{Supplementary Files}


This is a list of supplementary files associated with this preprint. Click to download.

- FigS1.png 